\title{
Lattice Boltzmann simulation of double-diffusive natural convection of viscoplastic fluids in a porous cavity
}

\author{
Cite as: Phys. Fluids 31, 013105 (2019); doi: 10.1063/1.5074089 \\ Submitted: 21 October 2018 - Accepted: 28 December 2018 • \\ Published Online: 17 January 2019
}

Gholamreza Kefayati

\section{AFFILIATIONS}

School of Engineering, University of Tasmania, Hobart, Tasmania, Australia

a) Electronic mail: gholamreza.kefayati@utas.edu.au

\begin{abstract}
In this paper, a two-dimensional double diffusive natural convection in a porous cavity filled with viscoplastic fluids is simulated. The dimensional and non-dimensional macroscopic equations are presented, employing the Papanastasiou model for viscoplastic fluids and the Darcy-Brinkman-Forchheimer model for porous media. An innovative approach based on a modification of the lattice Boltzmann method is explained and validated with previous studies. The effects of the pertinent dimensionless parameters are studied in different ranges. The extensive results of streamlines, isotherms, and isoconcentration contours, yielded/unyielded regions, and local and average Nusselt and Sherwood numbers are presented and discussed.
\end{abstract}

Published under license by AIP Publishing. https://doi.org/10.1063/1.5074089

\section{INTRODUCTION}

The lattice Boltzmann method (LBM) has been known as a powerful mesoscopic numerical method to model a broad variety of complex fluid flow phenomena. ${ }^{1-15}$ Huilgol and Kefayati $^{16}$ introduced an innovative LBM that can be applied to all fluids, whether they be Newtonian, or power law fluids, or viscoelastic and viscoplastic fluids. In fact, in this method, the equilibrium distribution function is altered in different directions and nodes while the relaxation time is fixed. Independency of the method to the relaxation time in contrast to common LBM provokes the method to solve different nonNewtonian fluids successfully as the method protects the positive points of LBM simultaneously. Following the previous study, Huilgol and Kefayati ${ }^{17}$ derived the two and three dimensional equations of this method for the Cartesian, cylindrical, and spherical coordinates. Recently, Kefayati et al. ${ }^{18}$ developed and improved the method for different thermal problems of non-Newtonian fluids through porous media. To validate the accuracy of the method, natural convection in a porous cavity was studied and compared with previous studies. In addition, in this study, natural convection of power-law and Bingham fluids in a porous cavity was simulated.
Flows in a porous enclosure driven by buoyancy force are a fundamental problem in fluid mechanics due to its importance in various industrial and technological applications such as microwave heating, geothermal systems, energy storage, gas transport, drying, and crystallization. ${ }^{19,20}$ This type of flow can be used as a benchmark in academic researches and various engineering applications. ${ }^{21-31}$

In the last three decades, there has been considerable attention to study the natural convection in the presence of the mass transfer in a square cavity through porous media due to a large number of technical applications such as packed sphere beds, chemical catalytic reactors, grain storage, solar heating, atmosphere study, geothermal reservoirs, solidification of casting, and crude oil production. So, the double diffusive natural convection in porous cavities has been simulated by many researchers. ${ }^{32-44}$

However, in the most cited industries which contain porous media, the fluid flow does not follow the classical Newtonian behavior. In some of the industries such as solidification of casting, oil recovery, food processing, and materials processing, the fluid flow shows viscoplastic behavior. In the case of the solidification of casting, double diffusive natural convection is the phenomenon that results from the 
effect of gravity on density differences caused by phase or compositional variations in the liquid. In most solidification models, the flow through the mushy zone is treated as flow through porous media. ${ }^{45}$ In addition, at temperatures between the liquidus and solidus, the metal is neither liquid nor solid and exhibits viscoplastic rheological properties. ${ }^{46}$ Viscoplastic fluids form a special sub-class of non-Newtonian fluids in which the flow field is divided into two regions: the first is an unyielded zone where the fluid is at rest or undergoes a rigid motion and the second where the fluid flows like a viscous liquid. In the unyielded zone, the stress is less than or equal to a critical value, the yield stress, and in the yielded region, the stress exceeds the yield stress. Natural convection of viscoplastic fluids in a cavity in the absence of the mass transfer has been studied in the last decade by some researchers numerically and experimentally. ${ }^{47-61}$

However, double diffusive natural convection (driven by cooperating thermal and solutal buoyancy forces) of viscoplastics in a cavity is limited to our recent investigations into this area. Kefayati ${ }^{62}$ simulated double-diffusive natural convection, studying Soret and Dufour effects and viscous dissipation in an inclined square cavity filled with Bingham fluid by LBM. Furthermore, entropy generations through fluid friction, heat transfer, and mass transfer were studied. The problem was solved by applying the regularised Papanastasiou model. Kefayati and Tang ${ }^{63}$ also studied double-diffusive natural convection of viscoplastic fluids and entropy generation in an open cavity, using the same approach.

The main aim of this study is to develop the introduced LBM and simulate double diffusive natural convection of a viscoplastic fluid in a porous cavity. The relevant macroscopic equations are listed in Sec. II, and the numerical method (LBM) is described in Sec. III. In Sec. IV, the present results are validated with previous numerical investigations and, in Sec. V, the effects of the main non-dimensional parameters on heat and mass transfer, fluid flow, and yielded/unyielded sections are examined.

\section{THEORETICAL FORMULATION}

Two-dimensional double diffusive natural convection in square enclosures is simulated where the enclosures are considered to be completely filled with a viscoplastic fluid obeying the Bingham model. The geometry of the present problem is shown in Fig. 1. The temperature and concentration of the left wall are considered to be maintained at high temperature and concentration of $\mathrm{T}_{\mathrm{H}}$ and $\mathrm{C}_{\mathrm{H}}$ as the right sidewall is kept at low temperature and concentration of $\mathrm{T}_{C}$ and $\mathrm{C}_{C}$. There is no heat generation and thermal radiation. The flow is steady, incompressible, and laminar. The density variation is approximated by the standard Boussinesq model for temperature and concentration. Viscous dissipation and Soret and Dufour effects are neglected.

Flows in porous media are usually modeled based on the volume-averaging at the representative elementary volume (REV) scale. In this approach, many models have been introduced in the literature, such as the Darcy, the DarcyBrinkman, and the Darcy-Brinkman-Forchheimer models. In

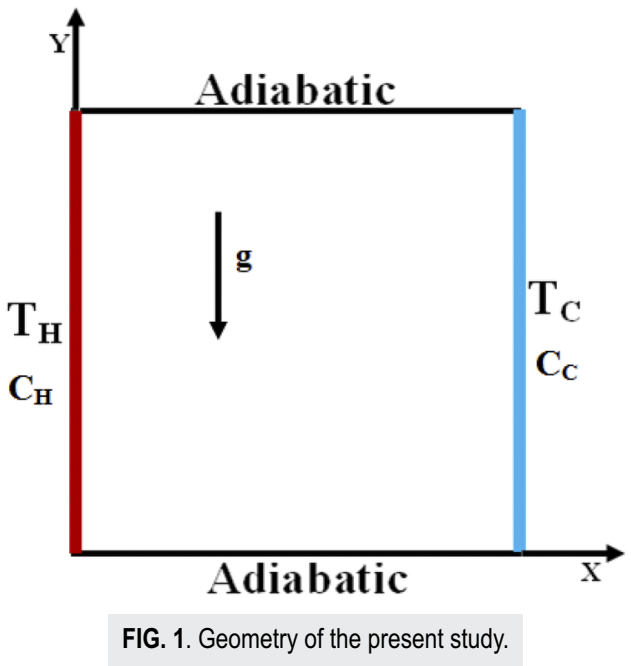

these models, fluid forces and the solid drag force are considered in the momentum equation. ${ }^{19,20}$ Here, the applied approach is the Darcy-Brinkman-Forchheimer model. The local thermal equilibrium is considered to be valid; i.e., the temperature of the solid phase and temperature of the fluid phase within the porous medium are equal. In a porous medium, the fluid flow mainly occurs through the pores or void space between the solid particles. Hence, either Darcy velocity or intrinsic velocity can be defined. The current work involves the intrinsic velocity based on the fluid control volume.

\section{A. Dimensional equations}

Based on the above assumptions, the dimensional mass, momentum, energy, and concentration equations $\operatorname{are}^{18,21,33-44,62,63}$

$$
\begin{gathered}
\frac{\partial u}{\partial x}+\frac{\partial v}{\partial y}=0 \\
\frac{\rho}{\epsilon^{2}}\left(u \frac{\partial u}{\partial x}+v \frac{\partial u}{\partial y}\right)=-\frac{1}{\epsilon} \frac{\partial(\epsilon p)}{\partial x}+\frac{1}{\epsilon}\left(\frac{\partial \tau_{x x}}{\partial x}+\frac{\partial \tau_{x y}}{\partial y}\right)-\Lambda_{x} \\
\frac{\rho}{\epsilon^{2}}\left(u \frac{\partial v}{\partial x}+v \frac{\partial v}{\partial y}\right)=-\frac{1}{\epsilon} \frac{\partial(\epsilon p)}{\partial y}+\frac{1}{\epsilon}\left(\frac{\partial \tau_{y y}}{\partial y}+\frac{\partial \tau_{x y}}{\partial x}\right) \\
+g \rho\left[\beta_{\mathrm{T}}\left(\mathrm{T}-\mathrm{T}_{\mathrm{C}}\right)+\beta_{\mathrm{C}}\left(\mathrm{C}-\mathrm{C}_{\mathrm{C}}\right)\right]-\Lambda_{y}
\end{gathered}
$$

Here, $x$ and $y$ are the coordinates varying along the horizontal and vertical directions, respectively; $u$ and $v$ are the velocity components in the $x$ and $y$ directions, respectively; $p$ is the dynamic pressure; and $\tau_{x x}, \tau_{x y}, \tau_{y y}$ are the stresses. $\rho$ and $g$ are the density and the gravity acceleration, respectively. $\beta_{\mathrm{T}}$ and $\beta_{\mathrm{C}}$ are the thermal and solutal expansion coefficients, respectively. $\boldsymbol{\Lambda}=\left(\Lambda_{x}, \Lambda_{y}\right)$ is the force term due to the porous medium and is expressed as

$$
\boldsymbol{\Lambda}=\left[\frac{\eta}{\mathrm{K}}+\frac{\rho \mathrm{E}_{\epsilon}}{\sqrt{\mathrm{K}}}|\mathbf{u}|\right] \mathbf{u}, \quad|\mathbf{u}|=\sqrt{u^{2}+v^{2}},
$$




$$
K=\frac{\epsilon^{3} d^{2}}{150(1-\epsilon)^{2}}, \quad E_{\epsilon}=\frac{1.75}{\sqrt{150 \epsilon^{3}}},
$$

where $\eta$ is the dynamic viscosity and $d$ is the solid particle diameter. $\mathrm{K}$ is the permeability of the porous medium, and $\epsilon$ is the porosity of the porous medium.

$$
u \frac{\partial \mathrm{T}}{\partial x}+v \frac{\partial \mathrm{T}}{\partial y}=\alpha_{e}\left(\frac{\partial^{2} \mathrm{~T}}{\partial x^{2}}+\frac{\partial^{2} \mathrm{~T}}{\partial y^{2}}\right),
$$

where $\mathrm{T}$ is the temperature and $\alpha_{e}$ is the effective thermal diffusivity of the porous medium as

$$
\alpha_{e}=\frac{k_{e}}{\left(\rho c_{p}\right)_{f}} \quad k_{e}=\epsilon k_{f}+(1-\epsilon) k_{s},
$$

where $k_{e}$ and $c_{p}$ are the effective thermal conductivity and the specific heat of the fluid at constant pressure, respectively.

$$
u \frac{\partial \mathrm{C}}{\partial x}+v \frac{\partial \mathrm{C}}{\partial y}=\mathrm{D}\left(\frac{\partial^{2} \mathrm{C}}{\partial x^{2}}+\frac{\partial^{2} \mathrm{C}}{\partial y^{2}}\right),
$$

where $\mathrm{C}$ is the concentration and $\mathrm{D}$ is the mass diffusivity.

\section{B. Constitutive model}

Bingham ${ }^{64}$ constituted the viscoplastic fluids as follows:

$$
\begin{gathered}
\begin{cases}\mathbf{A}_{1}=0, & |\boldsymbol{\tau}| \leq \tau_{y}, \\
\boldsymbol{\tau}=\left(\eta_{p}+\frac{\tau_{y}}{\dot{\gamma}}\right) \mathbf{A}_{1}, & |\boldsymbol{\tau}|>\tau_{y},\end{cases} \\
\mathbf{A}_{1}=\nabla \mathbf{u}+\nabla \mathbf{u}^{\mathrm{T}} .
\end{gathered}
$$

The plastic viscosity $\eta_{p}$ and the yield stress $\tau_{y}$ are constant, and the two invariants $\dot{\gamma}$ and $|\tau|$ are defined as follows:

$$
\dot{\gamma}=\frac{1}{\sqrt{2}} \sqrt{\mathbf{A}_{1}: \mathbf{A}_{1}}, \quad|\tau|=\frac{1}{\sqrt{2}} \sqrt{\tau: \tau}
$$

An alternative approach is to use the so-called regularization methods in which the constitutive equation modified by a regularized equation applies everywhere in the flow field in both yielded and unyielded regions such as the Papanastasiou ${ }^{65}$ model. In the Papanastasiou model, which is of interest here, the constitutive equation for the incompressible Bingham fluid is replaced by that of a material with a non-Newtonian viscosity. That is,

$$
\boldsymbol{\tau}=\eta(\dot{\gamma}) \mathbf{A}_{1}
$$

where

$$
\eta(\dot{\gamma})=\eta_{p}+\frac{\tau_{y}}{\dot{\gamma}}[1-\exp (-m \dot{\gamma})] .
$$

The material parameter, $m$, introduced here is to control exponential growth of the stress, and an ideal Bingham fluid can be closely approximated if $m$ is large enough. With consideration to previous studies, ${ }^{66}$ we fix the constant parameter at $m=10^{6}$.

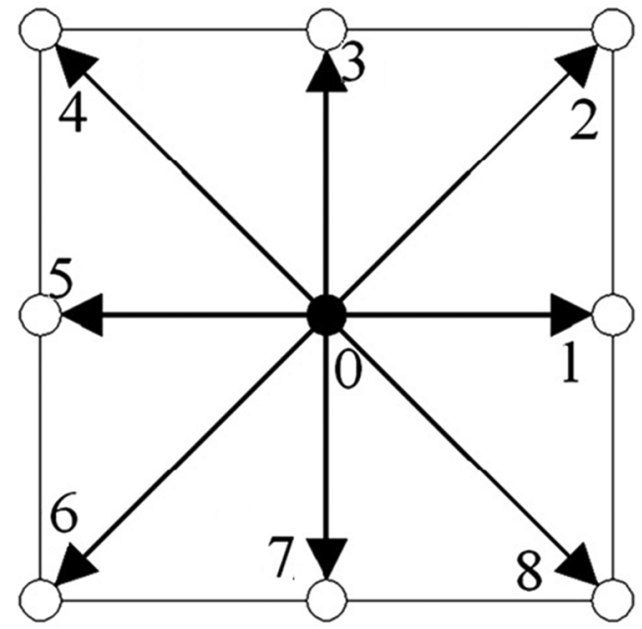

FIG. 2. Discrete velocity distribution in D2Q9.

\section{Boundary conditions}

The flow domain is given by $\Omega=(0, L) \times(0, L)$, and the boundary $\Gamma=\partial \Omega$. The union of four disjoint subsets is as follows:

$$
\begin{aligned}
& \Gamma_{1}=\{(x, y), x=0,0 \leq y \leq L\}, \\
& \Gamma_{2}=\{(x, y), x=L, 0 \leq y \leq L\}, \\
& \Gamma_{3}=\{(x, y), 0 \leq x \leq L, y=0\}, \\
& \Gamma_{4}=\{(x, y), 0 \leq x \leq L, y=L\} .
\end{aligned}
$$

The boundary condition for the velocity is straightforward

$$
\left.\mathbf{u}\right|_{\Gamma_{1}}=\left.\mathbf{u}\right|_{\Gamma_{2}}=\left.\mathbf{u}\right|_{\Gamma_{3}}=\left.\mathbf{u}\right|_{\Gamma_{4}}=0 .
$$

The boundary conditions of the temperature are

TABLE I. Comparison between present and previous studies for natural convection of Bingham fluids by the average Nusselt number at the hot wall in different Rayleigh numbers for $\mathrm{Pr}=1$.

\begin{tabular}{cccc}
\hline \hline \multicolumn{1}{c}{ Bn } & Reference 48 & Reference 54 & Present study \\
\hline $\mathrm{Ra}=10^{4}$ & & & \\
\hline $\mathrm{Bn}=0$ & 2.23 & 2.23 & 2.23 \\
$\mathrm{Bn}=0.5$ & 2.00 & 1.91 & 1.95 \\
$\mathrm{Bn}=1$ & 1.70 & 1.65 & 1.68 \\
$\mathrm{Bn}=1.5$ & 1.43 & 1.49 & 1.45 \\
$\mathrm{Bn}=2$ & 1.21 & 1.34 & 1.30 \\
$\mathrm{Bn}=2.5$ & 1.10 & 1.21 & 1.18 \\
$\mathrm{Bn}=3$ & 1.00 & 1.10 & 1.05 \\
\hline $\mathrm{Ra}=10^{5}$ & & & \\
\hline & & & \\
$\mathrm{Bn}=0$ & 4.60 & 4.60 & 3.60 \\
$\mathrm{Bn}=1$ & 3.89 & 3.89 & 3.88 \\
$\mathrm{Bn}=2$ & 3.45 & 3.46 & 3.12 \\
$\mathrm{Bn}=3$ & 2.95 & 3.16 & \\
\hline \hline
\end{tabular}


TABLE II. Comparison of the average Nusselt number for different Rayleigh and Darcy numbers at $\epsilon=0.4$ and $\operatorname{Pr}=1$.

\begin{tabular}{ccccccc}
\hline \hline & Ra & Reference 21 & Reference 22 & Reference 23 & Reference 24 & Present study \\
\hline $\mathrm{Da}=10^{-2}$ & & & & & & \\
\hline & $10^{3}$ & 1.010 & 1.008 & 1.007 & 1.008 & 1.008 \\
& $10^{4}$ & 1.408 & 1.367 & 1.362 & 1.365 & 1.366 \\
& $10^{5}$ & 2.983 & 2.998 & 3.009 & 3.012 & 3.010 \\
\hline $\mathrm{Da}=10^{-4}$ & & & & & \\
\hline & $10^{5}$ & 1.067 & 1.066 & 1.067 & 1.067 & 1.067 \\
& $10^{6}$ & 2.550 & 2.603 & 2.630 & 2.618 & 2.612 \\
& $10^{7}$ & 7.810 & 7.788 & 7.808 & 7.811 & 7.809 \\
\hline $\mathrm{Da}=10^{-6}$ & & & & & \\
\hline & $10^{7}$ & 1.79 & 1.077 & 1.085 & 1.089 & 1.081 \\
& $10^{8}$ & 2.970 & 2.955 & 2.949 & 3.014 & 2.998 \\
& $10^{9}$ & 11.460 & 11.395 & 11.610 & 11.733 & 11.512 \\
\hline \hline
\end{tabular}

$$
\left.\mathrm{T}\right|_{\Gamma_{1}}=\mathrm{T}_{\mathrm{H}},\left.\quad \mathrm{T}\right|_{\Gamma_{2}}=\mathrm{T}_{\mathrm{C}}, \quad \partial \mathrm{T} /\left.\partial y\right|_{\Gamma_{3}}=\partial \mathrm{T} /\left.\partial y\right|_{\Gamma_{4}}=0
$$

The boundary conditions of the concentration are

$$
\left.\mathrm{C}\right|_{\Gamma_{1}}=\mathrm{C}_{\mathrm{H}},\left.\quad \mathrm{C}\right|_{\Gamma_{2}}=\mathrm{C}_{\mathrm{C}}, \quad \partial \mathrm{C} /\left.\partial y\right|_{\Gamma_{3}}=\partial \mathrm{C} /\left.\partial y\right|_{\Gamma_{4}}=0
$$

\section{Non-dimensional equations}

The buoyancy velocity scale is $U=\left(\frac{\alpha_{e}}{L}\right) \mathrm{Ra}_{m}{ }^{0.5}$ as $\mathrm{Ra}_{m}$ is the modified Rayleigh number. In order to proceed to the numerical solution of the system, the following nondimensional variables are introduced where ${ }^{18,25,26,32-44,62,63}$

$$
\begin{gathered}
x^{*}=\frac{\mathbf{x}}{\mathrm{L}}, \quad \mathbf{u}^{*}=\frac{\mathbf{u}}{\mathrm{U}}, \quad p^{*}=\frac{p}{\rho \mathrm{U}^{2}}, \\
\mathrm{~T}^{*}=\left(\mathrm{T}-\mathrm{T}_{\mathrm{C}}\right) / \Delta \mathrm{T}, \quad \Delta \mathrm{T}=\mathrm{T}_{\mathrm{H}}-\mathrm{T}_{\mathrm{C}}, \\
\mathrm{C}^{*}=\left(\mathrm{C}-\mathrm{C}_{\mathrm{C}}\right) / \Delta \mathrm{C}, \quad \Delta \mathrm{C}=\mathrm{C}_{\mathrm{H}}-\mathrm{C}_{\mathrm{C}}, \\
\alpha_{e}=\frac{k_{e}}{\epsilon \rho c p_{f}}, \quad \mathrm{Ra}_{m}=\frac{\rho^{2} \beta_{\mathrm{T}} g \mathrm{~L}^{3} \Delta \mathrm{TPr} \mathrm{Pr}_{m}}{\eta_{p}^{2}}, \\
\operatorname{Pr}_{m}=\frac{\eta_{p}}{\alpha_{e} \rho}, \quad \mathrm{Da}_{m}=\frac{\mathrm{K}}{\epsilon \mathrm{L}^{2}}, \quad \mathrm{Bn}=\frac{\tau_{y} \mathrm{~L}}{\eta_{p} \mathrm{U}}, \quad \text { Le }=\frac{\alpha_{e}}{\mathrm{D}} .
\end{gathered}
$$

In other words, the relationship between the modified and actual Prandtl, Rayleigh, and Darcy numbers are $\mathrm{Pr}_{m}=\mathrm{Pr}$ $\times \epsilon, \mathrm{Ra}_{m}=\mathrm{Ra} \times \epsilon$, and $\mathrm{Da}_{m}=\mathrm{Da} / \epsilon$.

By substitution of Eqs. (18a)-(18e) in the dimensional equations and dropping the asterisks, the following system of non-dimensional mass, momentum, energy, and concentration equations is derived:

$$
\begin{gathered}
\frac{\partial u}{\partial x}+\frac{\partial v}{\partial y}=0 \\
u \frac{\partial u}{\partial x}+v \frac{\partial u}{\partial y}=-\frac{\partial p}{\partial x}+\frac{\operatorname{Pr}_{m}}{\sqrt{\mathrm{Ra}_{m}}}\left(\frac{\partial \tau_{x x}}{\partial x}+\frac{\partial \tau_{x y}}{\partial y}\right) \\
-\frac{\operatorname{Pr}_{m} u}{\mathrm{Da}_{m} \sqrt{\mathrm{Ra}_{m}}}-\frac{u|\mathbf{u}|}{\sqrt{\mathrm{Da}_{m}}} \frac{1.75}{\sqrt{150}}
\end{gathered}
$$

$$
\begin{aligned}
u \frac{\partial v}{\partial x}+v \frac{\partial v}{\partial y}= & -\frac{\partial p}{\partial y}+\frac{\operatorname{Pr}_{m}}{\sqrt{\mathrm{Ra}_{m}}}\left(\frac{\partial \tau_{y x}}{\partial x}+\frac{\partial \tau_{y y}}{\partial y}\right)+\operatorname{Pr}_{m}(\mathrm{~T}+\mathrm{NC}) \\
& -\frac{\operatorname{Pr}_{m} v}{\mathrm{Da}_{m} \sqrt{\mathrm{Ra}_{m}}}-\frac{v|\mathbf{u}|}{\sqrt{\mathrm{Da}_{m}}} \frac{1.75}{\sqrt{150}}
\end{aligned}
$$

Goyeau et al.
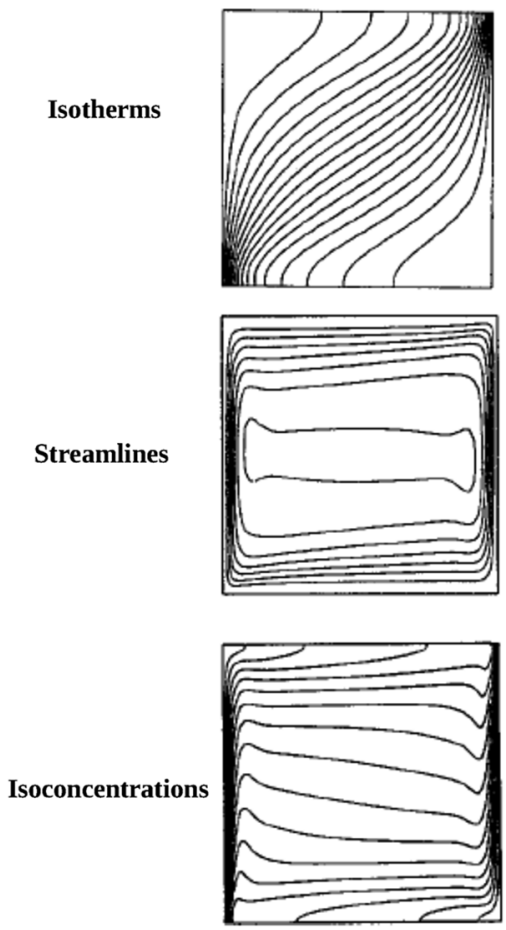

FIG. 3. Comparison of the isotherms, streamlines, and isoconcentrations between present study and the study of Goyeau et al. ${ }^{33}$ at $\mathrm{Le}=10, \mathrm{~N}=10, \mathrm{Da}=10^{-3}$, $\mathrm{Pr}=10$, and $R a^{*}=100\left(\mathrm{Ra}=R a^{*} / \mathrm{Da}=10^{5}\right)$ 
TABLE III. Grid independence study at $\mathrm{Ra}=10^{5}, \mathrm{Da}=10^{-3}, \epsilon=0.4, \mathrm{Bn}=1$, $\mathrm{N}=0.1, \mathrm{Le}=2.5$, and $\mathrm{Pr}=1$.

\begin{tabular}{llc}
\hline \hline Mesh size & $\mathrm{Nu}$ avg & $\mathrm{Sh}_{\text {avg }}$ \\
\hline $120^{*} 120$ & 2.5532 & 4.4820 \\
$140^{*} 140$ & 2.4162 & 4.4174 \\
$160^{*} 160$ & 2.3851 & 4.4022 \\
$180^{*} 180$ & 2.3681 & 4.3992 \\
$200^{*} 200$ & 2.3603 & 4.3985 \\
$220^{*} 220$ & 2.3603 & 4.3985 \\
\hline \hline
\end{tabular}

$$
\begin{gathered}
u \frac{\partial \mathrm{T}}{\partial x}+v \frac{\partial \mathrm{T}}{\partial y}=\frac{1}{\sqrt{\mathrm{Ra}_{m}}}\left(\frac{\partial^{2} \mathrm{~T}}{\partial x^{2}}+\frac{\partial^{2} \mathrm{~T}}{\partial y^{2}}\right) \\
u \frac{\partial \mathrm{C}}{\partial x}+v \frac{\partial \mathrm{C}}{\partial y}=\frac{1}{\mathrm{Le} \sqrt{\mathrm{Ra}_{m}}}\left(\frac{\partial^{2} \mathrm{C}}{\partial x^{2}}+\frac{\partial^{2} \mathrm{C}}{\partial y^{2}}\right) .
\end{gathered}
$$

In the case of the Papanastasiou model, 65 the nondimensional apparent viscosity is given by
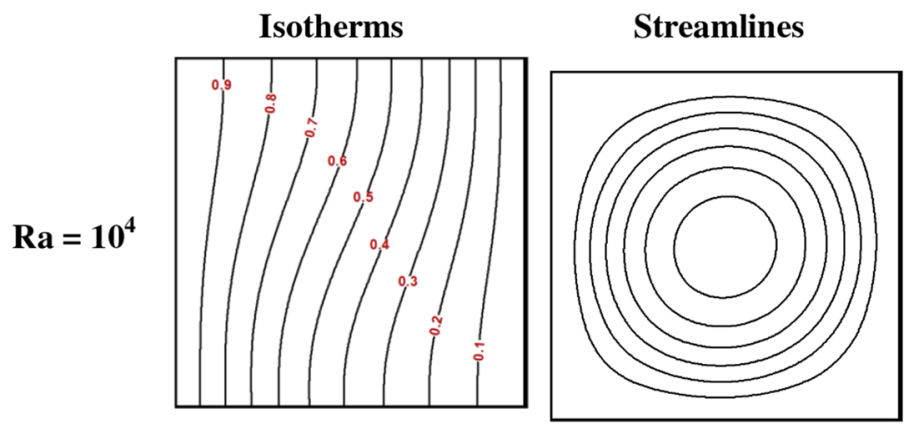

Isoconcentrations

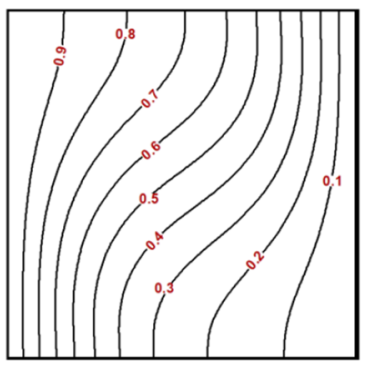

$$
\dot{\gamma}=\left\{2\left[\left(\frac{\partial u}{\partial x}\right)^{2}+\left(\frac{\partial v}{\partial y}\right)^{2}\right]+\left(\frac{\partial v}{\partial x}+\frac{\partial u}{\partial y}\right)^{2}\right\}^{\frac{1}{2}} .
$$

The local and the average Nusselt and Sherwood numbers on the hot wall are as follows:

$$
\mathrm{Nu}=\left(-\frac{\partial \mathrm{T}}{\partial x}\right)_{x=0}, \quad \mathrm{Sh}=\left(-\frac{\partial \mathrm{C}}{\partial x}\right)_{x=0},
$$

\section{Yielded/unyielded}

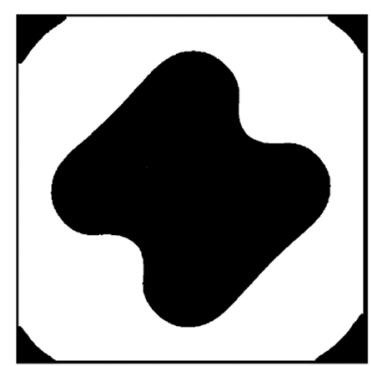

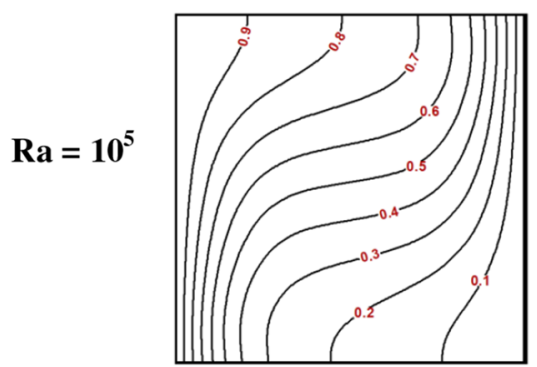
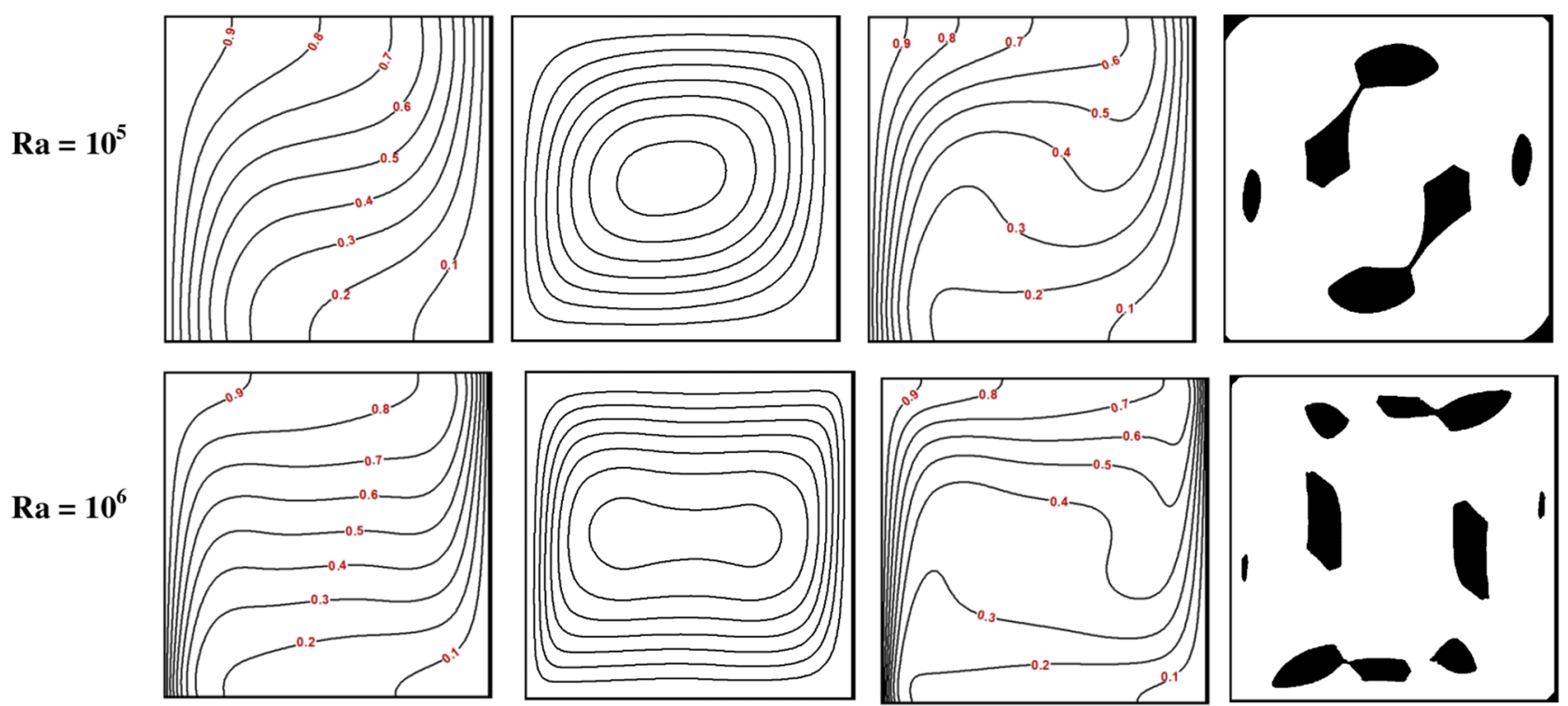

FIG. 4. The comparison of the isotherms, streamlines, isoconcentrations, and yielded/unyielded parts in different Rayleigh numbers at $B n=1, D a=10^{-3}, N=0.1, L e=2.5$, $\operatorname{Pr}=1$, and $\epsilon=0.4$. 

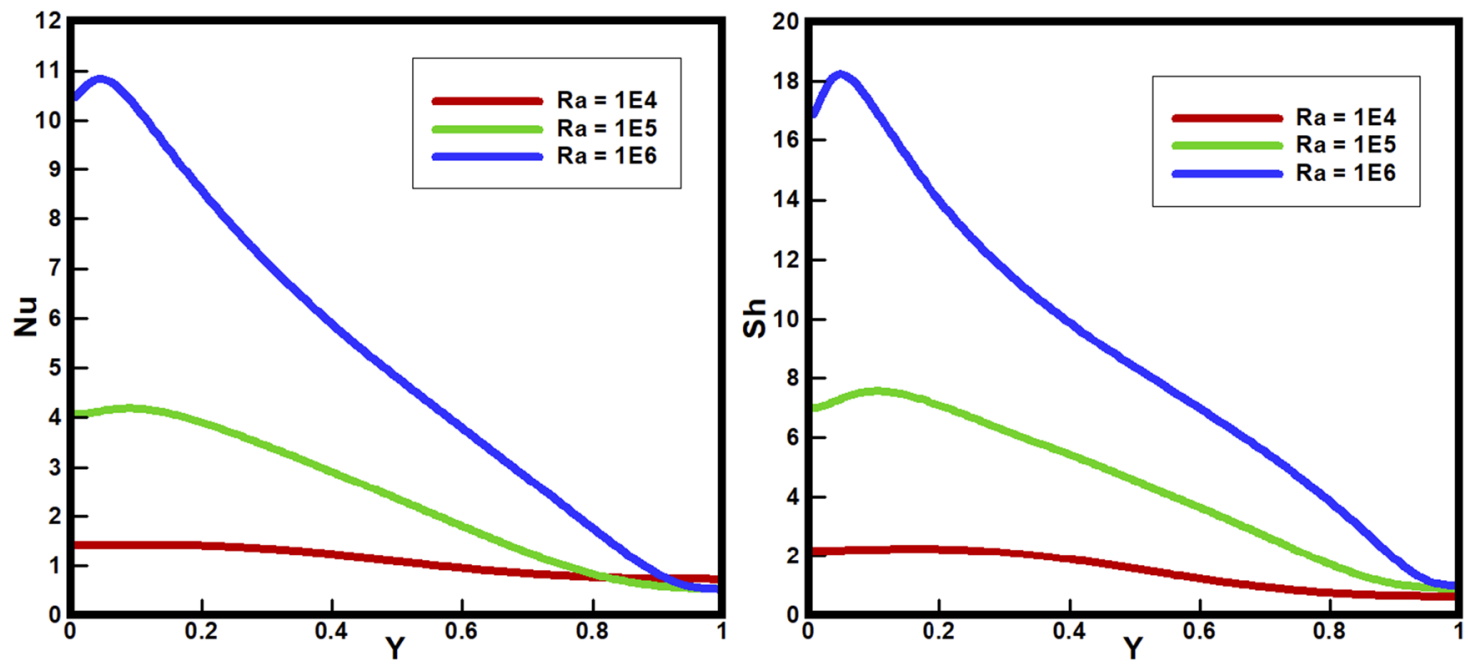

FIG. 5. The comparison of the local Nusselt and Sherwood numbers on the left wall in different Rayleigh numbers at $\mathrm{Bn}=1, \mathrm{Da}=10^{-3}, \mathrm{~N}=0.1, \mathrm{Le}=2.5, \mathrm{Pr}=1$, and $\epsilon=0.4$.

$$
N u_{a v g}=\int_{0}^{1} N u d x, \quad S h_{a v g}=\int_{0}^{1} \operatorname{Sh} d x .
$$

\section{LATTICE BOLTZMANN METHOD}

The approach is explained and approved in Refs. 16-18, so the method is described briefly here. For the continuity and momentum equations, a discrete particle distribution function $f_{\alpha}$ is defined as

$$
\frac{\partial f_{\alpha}}{\partial \mathrm{t}}+\boldsymbol{\xi}_{\alpha} \cdot \nabla_{\mathbf{x}} f_{\alpha}-\mathrm{F}_{\alpha}=-\frac{1}{\varepsilon \phi}\left(f_{\alpha}-f_{\alpha}^{e q}\right)
$$

where $\varepsilon$ is a small parameter to be prescribed when numerical simulations are considered. $\phi$ is the relaxation time. Associated with each node is a lattice velocity vector $\boldsymbol{\xi}_{\alpha}$, which is defined over a D2Q9 lattice (Fig. 2) as follows:

$$
\boldsymbol{\xi}_{\alpha}=\left\{\begin{array}{cc}
(0,0), & \alpha=0 \\
\sigma\left(\cos \Theta_{\alpha}, \sin \Theta_{\alpha}\right) & \alpha=1,3,5,7 \\
\sigma \sqrt{2}\left(\cos \Theta_{\alpha}, \sin \Theta_{\alpha}\right), & \alpha=2,4,6,8
\end{array}\right.
$$

Here, the angles $\Theta_{\alpha}$ are defined through $\Theta_{\alpha}=(\alpha-1) \pi / 4, \alpha=1$, $\ldots, 8$. The constant $\sigma$ has to be chosen with care for it affects numerical stability. The equilibrium distribution function, $f_{\alpha}^{e q}$, is ${ }^{16,17}$

$$
f_{\alpha}^{e q}=\mathrm{A}_{\alpha}+\boldsymbol{\xi}_{\alpha} \cdot \mathbf{B}_{\alpha}+\left(\boldsymbol{\xi}_{\alpha} \otimes \boldsymbol{\xi}_{\alpha}\right): \mathbf{C}_{\alpha}, \alpha=0,1,2, \ldots, 8 .
$$

The relation between the above parameters and nondimensional macroscopic values are as follows:

$$
\begin{gathered}
\mathrm{A}_{0}=\rho-\frac{2 p}{\sigma^{2}}-\frac{\rho|\mathbf{u}|^{2}}{\sigma^{2}}, \quad \mathrm{~A}_{\alpha}=0, \alpha=1,2, \ldots, 8 . \\
\mathbf{B}_{1}=\frac{\rho \mathbf{u}}{2 \sigma^{2}}=\mathbf{B}_{\alpha}, \alpha=1,3,5,7 ; \quad \mathbf{B}_{\alpha}=0, \alpha=0,2,4,6,8 .
\end{gathered}
$$

Next, the matrices $\mathbf{C}_{\alpha}$ are such that $\mathbf{C}_{0}=0 ; \mathbf{C}_{1}=\mathbf{C}_{\alpha}, \alpha=1$, $3,5,7 ; \mathbf{C}_{2}=\mathbf{C}_{\alpha}, \alpha=2,4,6,8$, where

$$
\begin{gathered}
\mathbf{C}_{1}=\left[\begin{array}{cc}
C_{11} & 0 \\
0 & C_{22}
\end{array}\right], \quad C_{11}=\frac{1}{2 \sigma^{4}}\left(p+\rho u^{2}-\frac{\operatorname{Pr}_{m}}{\sqrt{\mathrm{Ra}_{m}}} \tau_{x x}\right), \\
C_{22}=\frac{1}{2 \sigma^{4}}\left(p+\rho v^{2}-\frac{\operatorname{Pr}_{m}}{\sqrt{\mathrm{Ra}_{m}}} \tau_{y y}\right), \\
\mathbf{C}_{2}=\left[\begin{array}{cc}
0 & C_{12} \\
C_{21} & 0
\end{array}\right], \quad C_{12}=C_{21}=\frac{1}{8 \sigma^{4}}\left(\rho u v-\frac{\operatorname{Pr}_{m}}{\sqrt{\mathrm{Ra}_{m}}} \tau_{x y}\right) .
\end{gathered}
$$

The body force term $\mathrm{F}_{\alpha}$ can be defined as

$$
\begin{gathered}
\mathrm{F}_{\alpha}=0, \quad \alpha=0,2,4,6,8, \\
\mathrm{~F}_{\alpha}=\frac{1}{2 \sigma^{2}} \mathbf{S} \cdot \boldsymbol{\xi}_{\alpha}, \quad \alpha=1,3,5,7,
\end{gathered}
$$

where

$$
\begin{aligned}
\mathbf{S}= & {\left[-\frac{\operatorname{Pr}_{m} u}{\mathrm{Da}_{m} \sqrt{\mathrm{Ra}_{m}}}-\frac{u|\mathbf{u}|}{\sqrt{\mathrm{Da}_{m}}} \frac{1.75}{\sqrt{150}}\right] \mathbf{i} } \\
& +\left[\operatorname{Pr}_{m}(\mathrm{~T}+\mathrm{NC})-\frac{\operatorname{Pr}_{m} v}{\mathrm{Da}_{m} \sqrt{\mathrm{Ra}_{m}}}-\frac{v|\mathbf{u}|}{\sqrt{\mathrm{Da}_{m}}} \frac{1.75}{\sqrt{150}}\right] \mathbf{j}
\end{aligned}
$$

The internal energy distribution function $g_{\alpha}$ over a D2Q9 lattice is as follows:

$$
\frac{\partial g_{\alpha}}{\partial \mathrm{t}}+\xi_{\alpha} \cdot \nabla_{\mathbf{x}} g_{\alpha}-\mathrm{G}_{\alpha}=-\frac{1}{\varepsilon \phi}\left(g_{\alpha}-g_{\alpha}^{e q}\right) .
$$

TABLE IV. Comparison of the average Nusselt and Sherwood numbers for different Rayleigh numbers at $\epsilon=0.4, \mathrm{Bn}=1, \mathrm{~N}=0.1, \mathrm{Le}=2.5, \mathrm{Da}=10^{-3}$, and $\mathrm{Pr}=1$.

\begin{tabular}{lccc}
\hline \hline & $\mathrm{Ra}=10^{4}$ & $\mathrm{Ra}=10^{5}$ & $\mathrm{Ra}=10^{6}$ \\
\hline $\mathrm{Nu}_{\text {avg }}$ & 1.0852 & 2.3603 & 5.1512 \\
$\mathrm{Sh}_{\text {avg }}$ & 1.5068 & 4.3985 & 8.8681 \\
\hline \hline
\end{tabular}


Isotherms
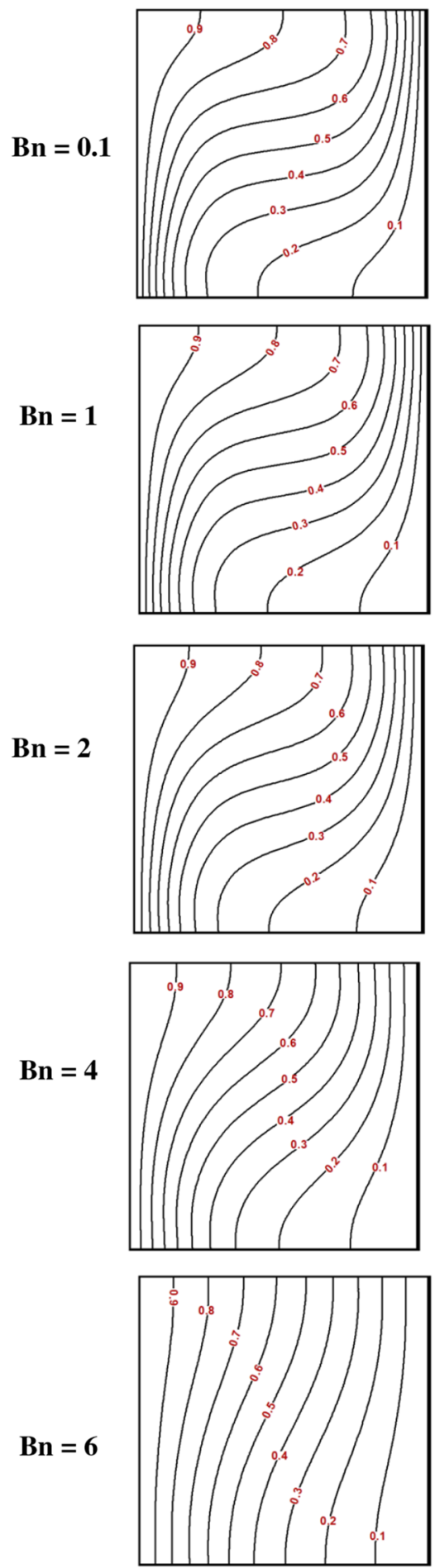

Streamlines
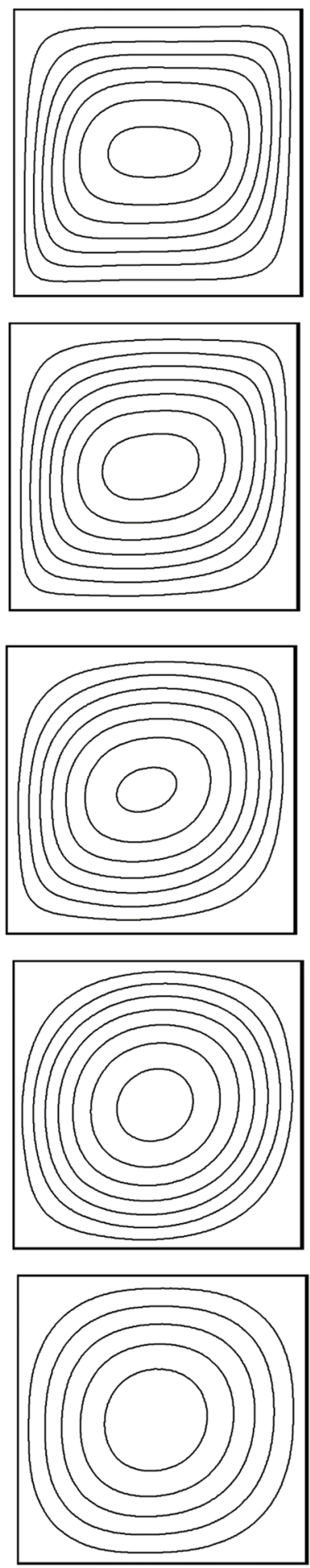

Isoconcentrations
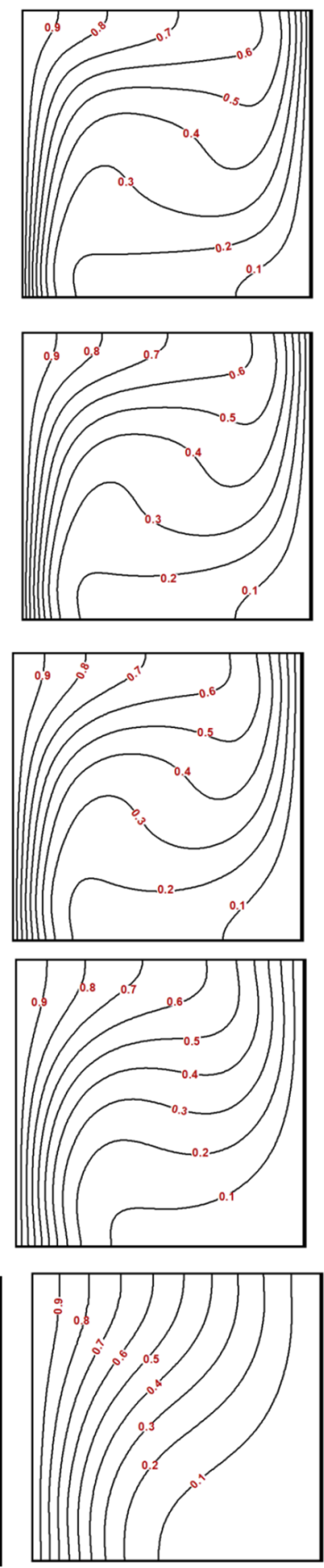

Yielded/unyielded
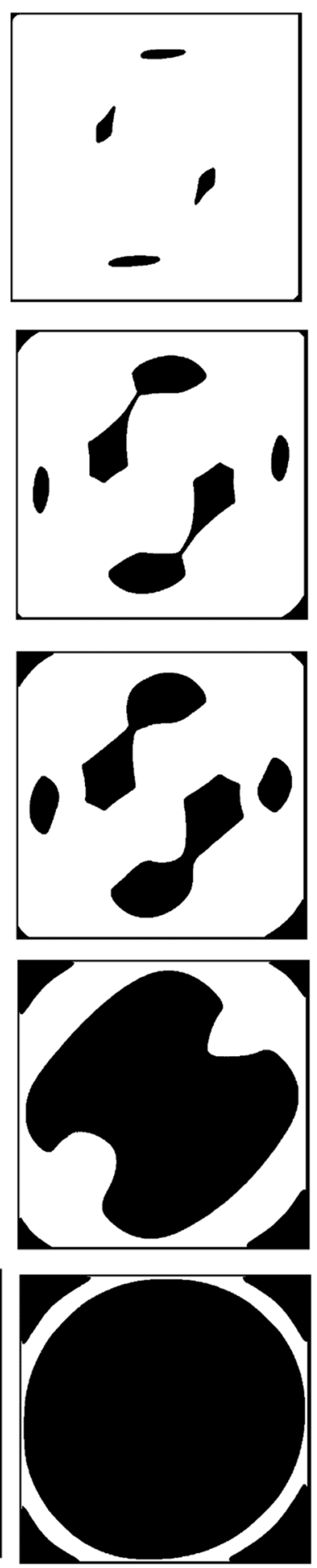

FIG. 6. The comparison of the isotherms, streamlines, isoconcentrations, and yielded/unyielded parts in different Bingham numbers at Ra $=10^{5}, \mathrm{Da}=10^{-3}, \mathrm{~N}=0.1$, $\mathrm{Le}=2.5, \operatorname{Pr}=1$, and $\epsilon=0.4$. 

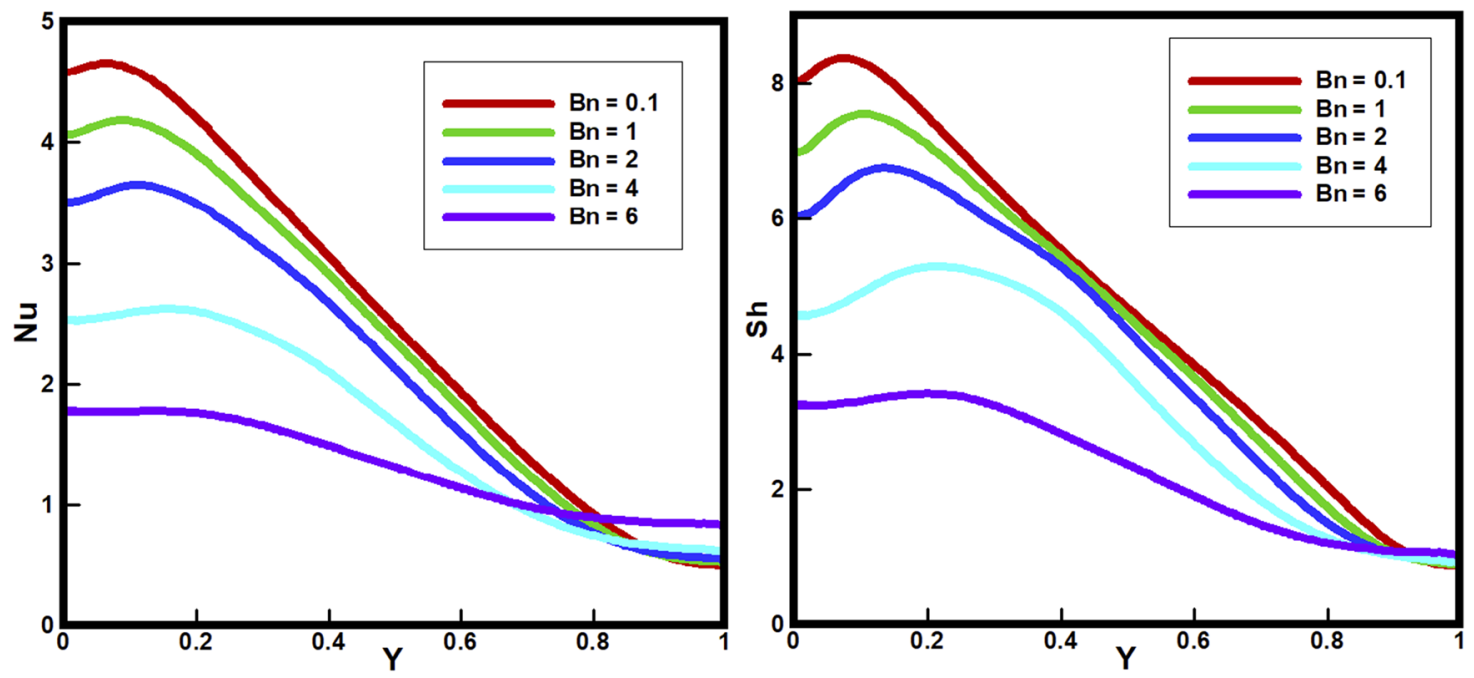

FIG. 7. The comparison of the local Nusselt and Sherwood numbers on the left wall in different Bingham numbers at $\operatorname{Ra}=10^{5}, \mathrm{Da}=10^{-3}, \mathrm{~N}=0.1, \mathrm{Le}=2.5, \mathrm{Pr}=1$, and $\epsilon=0.4$.

$g_{\alpha}^{e q}$ has a monomial expansion ${ }^{62,63}$

$$
g_{\alpha}^{e q}=\mathrm{D}_{\alpha}+\boldsymbol{\xi}_{\alpha} \cdot \mathbf{E}_{\alpha} .
$$

One way of satisfying the above is to assume, as before, that the scalars are given by $\mathrm{D}_{\alpha}=\mathrm{D}_{1}, \alpha=1,3,5,7$, and $\mathrm{D}_{\alpha}=\mathrm{D}_{2}$, $\alpha=2,4,6,8$. In this problem, the non-dimensional parameters are obtained as follows:

$$
\mathrm{D}_{0}=\mathrm{T}, \quad \mathrm{D}_{1}=0, \quad \mathrm{D}_{2}=0 .
$$

Regarding the vectors, it is assumed that $\mathbf{E}_{\alpha}=\mathbf{E}_{1}, \alpha=1,3,5,7$; $\mathbf{E}_{\alpha}=\mathbf{E}_{2}=0, \alpha=0,2,4,6,8$, where

$$
\mathbf{E}_{1}=\frac{1}{2 \sigma^{2}}\left(\mathbf{u} \mathrm{T}-\frac{1}{\sqrt{\mathrm{Ra}_{m}}} \frac{\partial \mathrm{T}}{\partial \mathbf{x}}\right)
$$

The internal concentration distribution function $h_{\alpha}$ over a D2Q9 lattice is

$$
\frac{\partial h_{\alpha}}{\partial \mathrm{t}}+\xi_{\alpha} \cdot \nabla_{\mathbf{x}} h_{\alpha}-H_{\alpha}=-\frac{1}{\varepsilon \phi}\left(h_{\alpha}-h_{\alpha}^{e q}\right) .
$$

Here, $h_{\alpha}^{e q}$ has a monomial expansion ${ }^{62,63}$

$$
h_{\alpha}^{e q}=M_{\alpha}+\xi_{\alpha} \cdot \mathbf{Z}_{\alpha} .
$$

One way of satisfying the above is to assume, as before, that the scalars are given by $M_{\alpha}=M_{1}, \alpha=1,3,5,7$, and $M_{\alpha}=M_{2}$, $\alpha=2,4,6,8$. In this problem, the non-dimensional parameters are obtained as follows:

$$
M_{0}=C, \quad M_{1}=0, \quad M_{2}=0 .
$$

Regarding the vectors, it is assumed that $\mathbf{Z}_{0}=0, \mathbf{Z}_{\alpha}=\mathbf{Z}_{1}, \alpha=1$, $3,5,7 ; \mathbf{Z}_{\alpha}=\mathbf{Z}_{2}, \alpha=2,4,6,8$, where

$$
\mathbf{Z}_{1}=\frac{1}{2 \sigma^{2}}\left(\mathbf{u} C-\frac{1}{\operatorname{Le} \sqrt{\operatorname{Ra}_{m}}} \frac{\partial C}{\partial \mathbf{x}}\right) .
$$

It should be noted that the source terms in the energy and concentration part are $G_{\alpha}=H_{\alpha}=0$.

\section{CODE VALIDATION AND GRID INDEPENDENCE}

The LBM scheme is utilized to simulate laminar double diffusive natural convection in a porous enclosure filled with a viscoplastic fluid. This problem is investigated at different parameters of the Rayleigh number $\left(\mathrm{Ra}=10^{4}-10^{6}\right)$, Darcy number $\left(\mathrm{Da}=10^{-2}\right.$ to $\left.10^{-6}\right)$, porosity $(\epsilon=0.1-0.9)$, Lewis number $(\mathrm{Le}=2.5,5$, and 10), Buoyancy ratio $(\mathrm{N}=-1,0.1,1$, and 10), Prandtl number $(\operatorname{Pr}=1,10,20,40$, and 100), and different Bingham numbers. In this study, isotherms, streamlines, isoconcentrations, and yielded/unyielded zones have been analyzed. The yielded and unyielded parts have been specified by white and black in the contour; respectively. The results of a Bingham fluid undergoing natural convection in an enclosure have been compared with the work of Turan et al. ${ }^{48}$ and Huilgol and Kefayati ${ }^{54}$ in Table I, which demonstrates good agreement. In addition, the accuracy of the employed numerical method in natural convection of the Bingham method was studied by the author in the previous study. ${ }^{58-60}$ The applied code for natural convection in a porous cavity is validated by the average Nusselt number on the hot wall with the previous studies of Nithiarasu et al., ${ }^{21}$ Guo and Zhao, ${ }^{22} \mathrm{Liu}$ et al., ${ }^{23}$ and Wang et al. ${ }^{24}$ in Table II in different Rayleigh and Darcy numbers at $\operatorname{Pr}=1$. The present approach is applied for

TABLE V. Comparison of the average Nusselt and Sherwood numbers for different Bingham numbers at $\epsilon=0.4, \mathrm{Ra}=10^{5}, \mathrm{~N}=0.1, \mathrm{Le}=2.5, \mathrm{Da}=10^{-3}$, and $\mathrm{Pr}=1$.

\begin{tabular}{lcccccc}
\hline \hline & $\mathrm{Bn}=0.1$ & $\mathrm{Bn}=1$ & $\mathrm{Bn}=2$ & $\mathrm{Bn}=4$ & $\mathrm{Bn}=6$ & $\mathrm{Bn}=8$ \\
\hline $\mathrm{Nu}$ avg & 2.5433 & 2.3603 & 2.1223 & 1.6620 & 1.3211 & 1.2462 \\
$\mathrm{Sh}_{\text {avg }}$ & 4.7075 & 4.3985 & 4.0563 & 3.3177 & 2.2985 & 1.9973 \\
\hline \hline
\end{tabular}



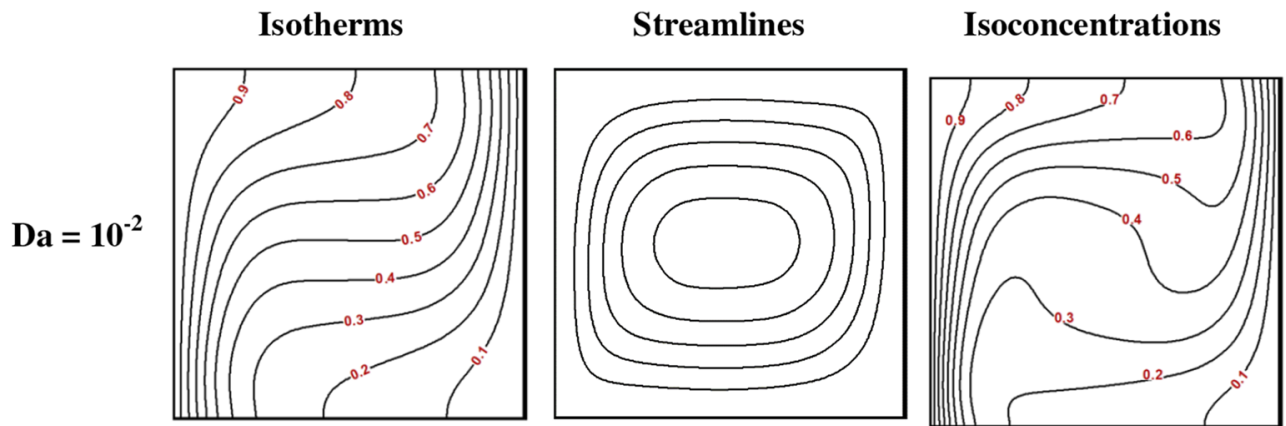

Yielded/unyielded
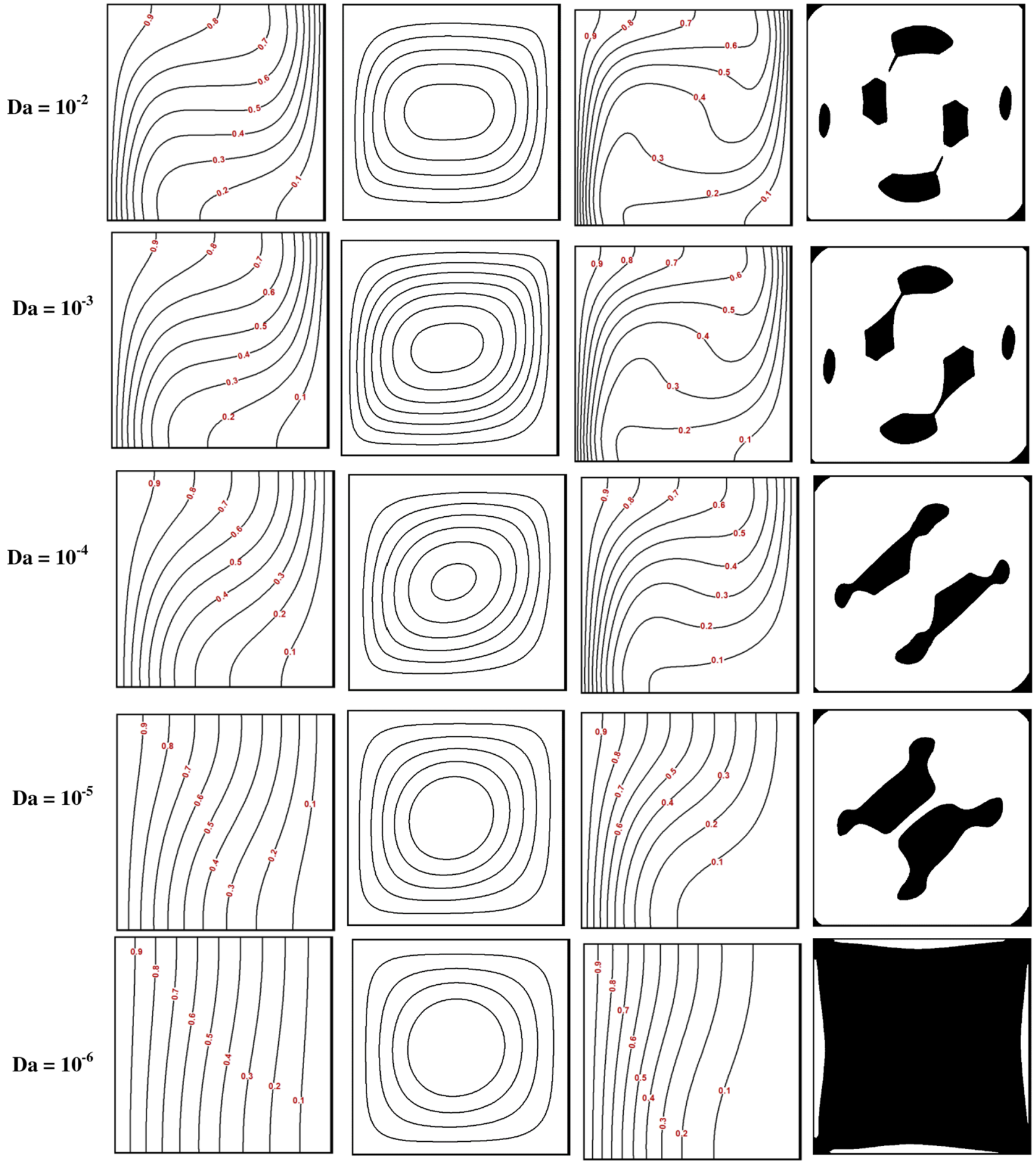

FIG. 8. The comparison of the isotherms, streamlines, isoconcentrations, and yielded/unyielded parts in different Darcy numbers at $\mathrm{Bn}=1, \mathrm{Ra}=10^{5}, \mathrm{~N}=0.1, \mathrm{Le}=2.5$, $\operatorname{Pr}=1$, and $\epsilon=0.4$. 

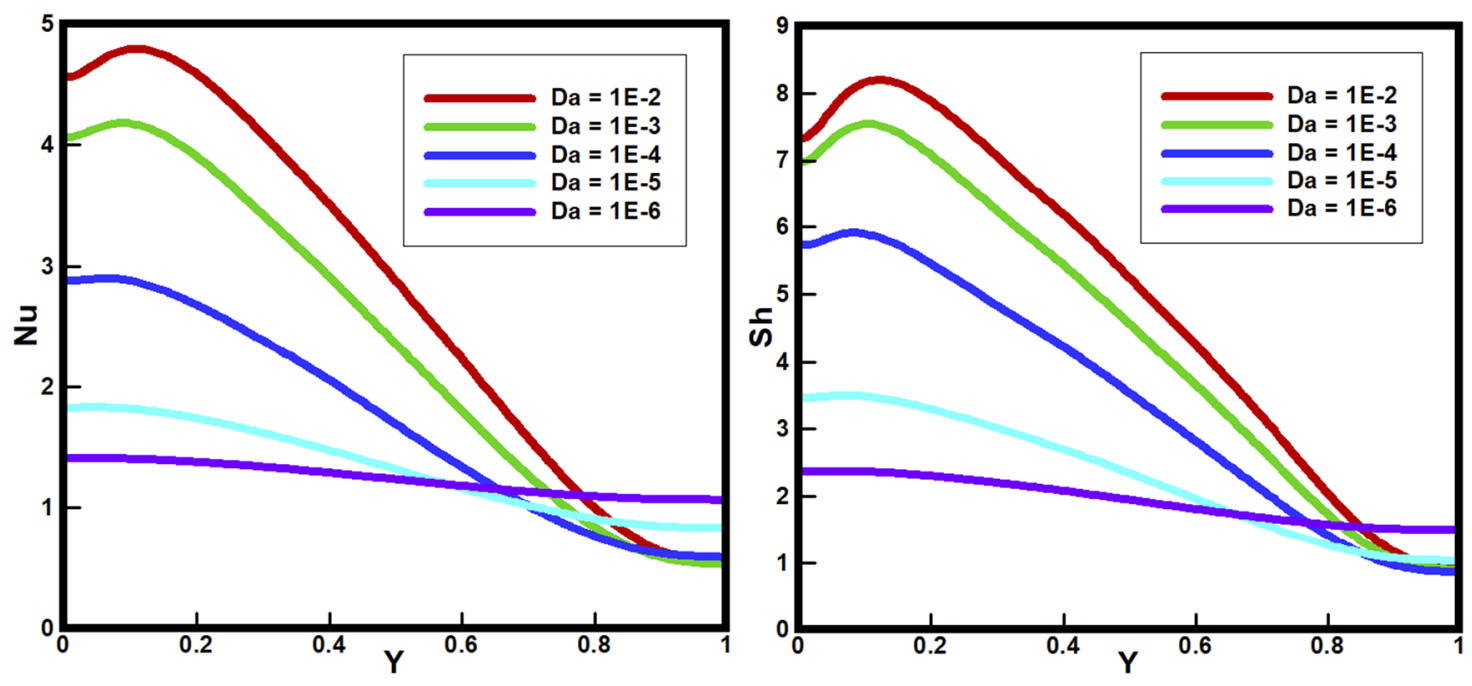

FIG. 9. The local Nusselt and Sherwood numbers on the hot wall in different Darcy numbers at $\mathrm{Ra}=10^{5}, \epsilon=0.4, \mathrm{~N}=0.1, \mathrm{Bn}=1, \mathrm{Le}=2.5$, and $\mathrm{Pr}=1$.

double diffusive natural convection of Bingham fluids in a cavity recently, 22,63 which demonstrates the accuracy of the utilized code for non-Newtonian Bingham fluids on double diffusive natural convection properly. The applied numerical method for double diffusive natural convection of porous media has been compared with the results of Goyeau et al. ${ }^{33}$ for $\mathrm{Le}=10, \mathrm{~N}=10, \mathrm{Da}=10^{-3}, \mathrm{Pr}=10$, and $\mathrm{Ra}^{*}=100(\mathrm{Ra}$ $\left.=\mathrm{R} a^{*} / \mathrm{Da}=10^{5}\right)$ in Fig. 3 , where the isotherms, streamlines, and isoconcentrations demonstrate the accuracy of the utilized code. An extensive mesh testing procedure was conducted to guarantee a grid independent solution. Six different mesh combinations were explored for the case of $\mathrm{Ra}=10^{5}, \mathrm{Da}=10^{-3}$, $\epsilon=0.4, \operatorname{Pr}=1, \mathrm{Le}=2.5$, and $\mathrm{Bn}=2$. The average Nusselt and Sherwood numbers on the hot wall have been studied. It was confirmed that the grid size $(200 \times 200)$ ensures a grid independent solution as portrayed in Table III.

TABLE VI. Comparison of the average Nusselt and Sherwood numbers for different porosities and Darcy numbers at $\mathrm{Bn}=1$, $\mathrm{Ra}=10^{5}, \mathrm{~N}=0.1, \mathrm{Le}=2.5$, and $\mathrm{Pr}=1$.

\begin{tabular}{|c|c|c|c|c|c|c|}
\hline & $\epsilon=0.1$ & $\epsilon=0.2$ & $\epsilon=0.4$ & $\epsilon=0.6$ & $\epsilon=0.8$ & $\epsilon=0.9$ \\
\hline \multicolumn{7}{|c|}{$\mathrm{Da}=10^{-2}$} \\
\hline$N u_{a v g}$ & 1.5571 & 2.0946 & 2.7889 & 3.2846 & 3.6729 & 3.8410 \\
\hline$S h_{\text {avg }}$ & 2.7554 & 3.7182 & 4.9336 & 5.7855 & 6.4404 & 6.7213 \\
\hline \multicolumn{7}{|c|}{$\mathrm{Da}=10^{-3}$} \\
\hline $\mathrm{N} u_{\text {ava }}$ & 1.3953 & 1.8184 & 2.3603 & 2.7309 & 3.0041 & 3.1204 \\
\hline $\mathrm{Sh}_{\text {avg }}$ & 2.5006 & 3.3703 & 4.3985 & 5.1057 & 5.6482 & 5.8829 \\
\hline \multicolumn{7}{|c|}{$\mathrm{Da}=10^{-4}$} \\
\hline$N u_{a v g}$ & 1.18884 & 1.4064 & 1.7190 & 1.9537 & 2.1307 & 2.2068 \\
\hline $\mathrm{Sh}_{\text {avg }}$ & 2.0188 & 2.7023 & 3.4555 & 3.9239 & 4.2303 & 4.3518 \\
\hline \multicolumn{7}{|c|}{$\mathrm{Da}=10^{-5}$} \\
\hline$N u_{a v g}$ & 1.0642 & 1.1559 & 1.3240 & 1.4620 & 1.5709 & 1.6181 \\
\hline $\mathrm{Sh}_{\text {avg }}$ & 1.5318 & 1.8928 & 2.3006 & 2.5599 & 2.7368 & 2.8095 \\
\hline \multicolumn{7}{|c|}{$\mathrm{Da}=10^{-6}$} \\
\hline$N u_{\text {avg }}$ & 1.0335 & 1.0999 & 1.2354 & 1.3490 & 1.4427 & 1.4840 \\
\hline $\mathrm{Sh}_{\text {avg }}$ & 1.3734 & 1.6306 & 1.9416 & 2.1486 & 2.3049 & 2.3717 \\
\hline
\end{tabular}




\section{RESULTS AND DISCUSSION}

\section{A. Rayleigh number effects}

The Rayleigh number is defined as the ratio of the buoyancy forces to the viscous forces. The effects of buoyancy force strengthen relative to the viscous force with increasing Rayleigh number for a given set of values of other parameters, which in turn augments heat and mass transfer by convection due to stronger buoyancy-driven flow with higher vertical velocity magnitude. Figure 4 shows the isotherms, streamlines, isoconcentrations, and yielded/unyielded parts in different Rayleigh numbers at $\mathrm{Bn}=1, \mathrm{Da}=10^{-3}, \mathrm{~N}=0.1, \mathrm{Le}=2.5, \mathrm{Pr}=1$, and $\epsilon=0.4$. The movements of the isotherms between the cold and hot walls ameliorate significantly, and they become progressively curved. Moreover, the gradient of temperature on the hot wall augments with the rise in Rayleigh number. In fact, it occurs while the thermal boundary layer thickness on the side walls decreases with increasing Rayleigh number. The streamlines exhibit that the convection process has been enhanced by the growth of Rayleigh numbers as the core of

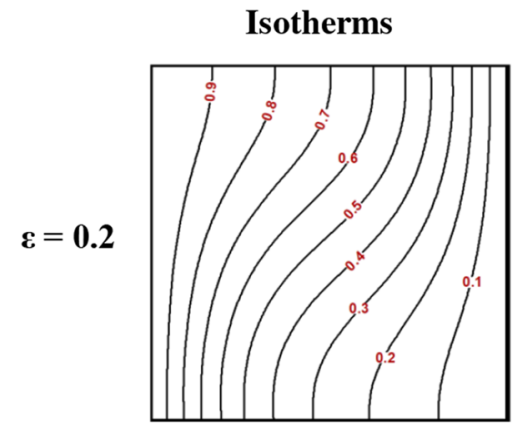

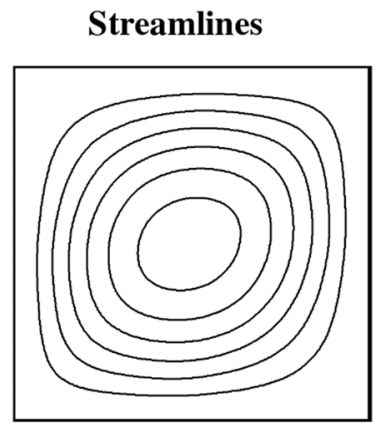

Isoconcentrations
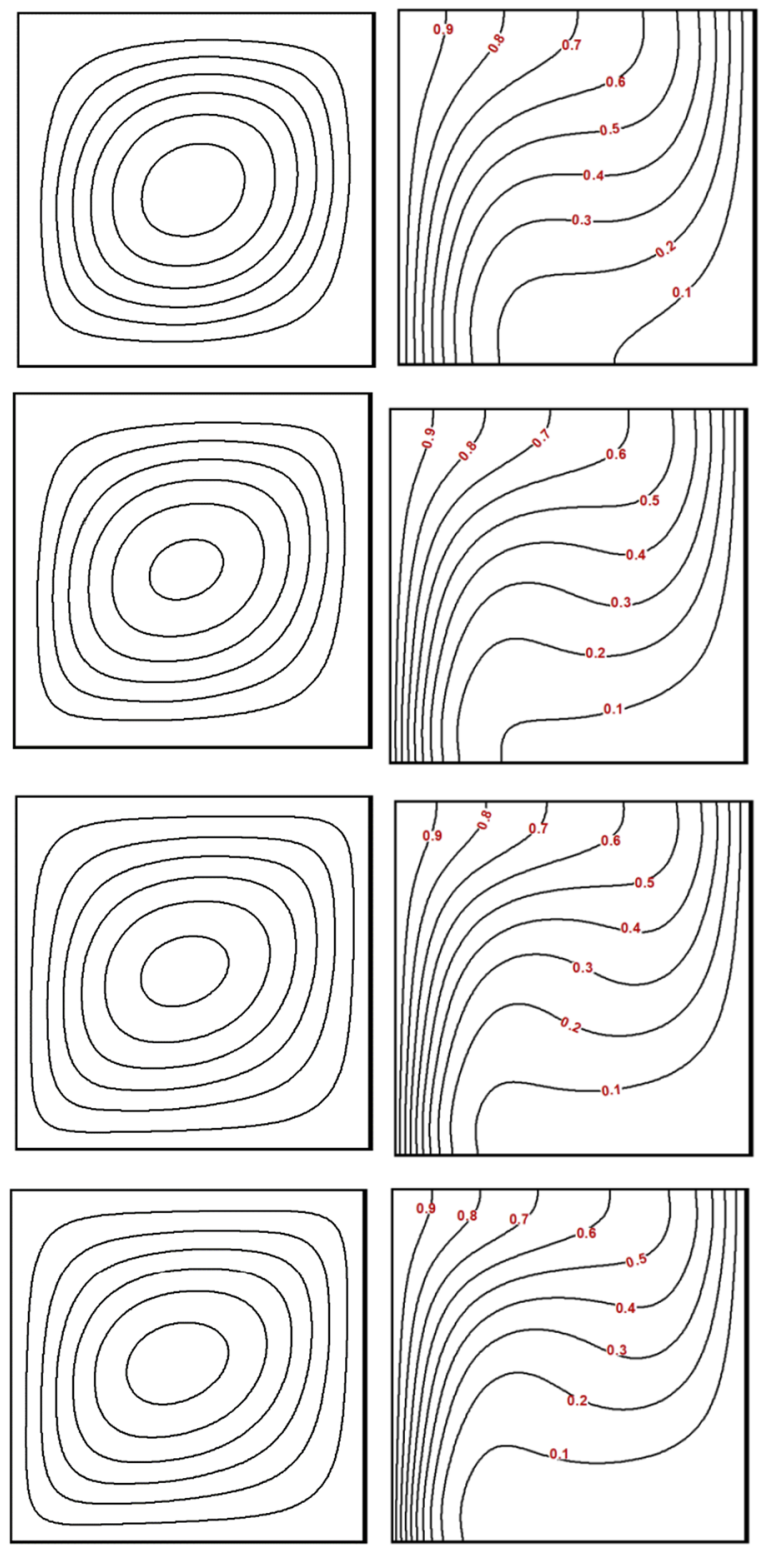

Yielded/unyielded
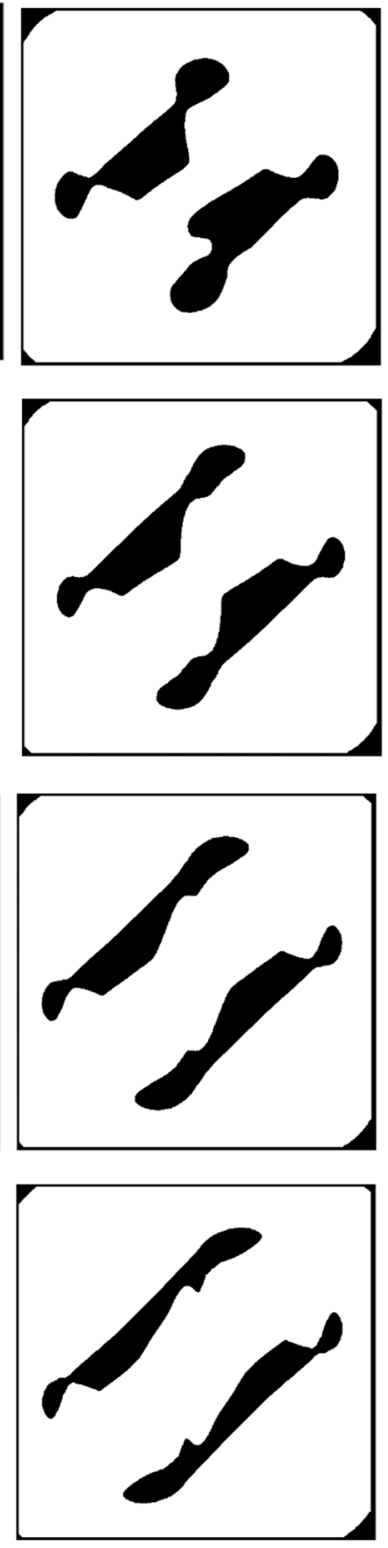

FIG. 10. The comparison of the isotherms, streamlines, and yielded/unyielded parts in different porosities at $\mathrm{Da}=10^{-4}, \mathrm{Ra}=10^{5}, \mathrm{~N}=0.1, \mathrm{Bn}=1, \mathrm{Pr}=1$, and $\mathrm{Le}=2.5$. 
the streamline changes and the streamlines traverse more distance in the cavity. The last column displays the yielded (white) and unyielded (black) regions for the studied Rayleigh numbers. It is clear that the proportion of the unyielded sections in the enclosure has enhanced with the drop in Rayleigh numbers markedly. Therefore, for a constant Bingham number, the increase in Rayleigh number causes the unyielded zones to decline. The isoconcentrations demonstrate the same manner where the increase in Rayleigh number enhances the gradient of isoconcentrations on the hot walls. It depicts that mass transfer increases significantly with the rise in Rayleigh number.

Figure 5 demonstrates the local Nusselt and Sherwood numbers on the left wall in different Rayleigh numbers at $\mathrm{Bn}=1, \mathrm{Da}=10^{-3}, \mathrm{~N}=0.1, \mathrm{Le}=2.5, \mathrm{Pr}=1$, and $\epsilon=0.4$. The local Nusselt and Sherwood numbers rise considerably as the Rayleigh number increases. Table IV indicates the average Nusselt and Sherwood numbers in different Rayleigh numbers at $\mathrm{Bn}=1, \mathrm{Da}=10^{-3}, \mathrm{~N}=0.1$, Le $=2.5$, and $\epsilon=0.4$. It, clearly, demonstrates that the average Nusselt and Sherwood numbers enhance as the Rayleigh number increases.

\section{B. Bingham number effects}

The Bingham number is the ratio of the yield stress of the fluid to the viscous stress caused by flow. Increasing values of this parameter suggest that the flow domain consists of predominantly unyielded regions, thereby suppressing convective transport of heat and mass transfer. Figure 6 illustrates the isotherms, streamlines, isoconcentrations, and yielded/unyielded parts in different Bingham numbers at $\mathrm{Ra}$ $=10^{5}, \mathrm{Da}=10^{-3}, \mathrm{~N}=0.1, \mathrm{Le}=2.5, \mathrm{Pr}=1$, and $\epsilon=0.4$. It shows that the curved shapes of the isotherm and isoconcentrations decline with the rise in Bingham number. The process causes the gradient of temperature and isoconcentration on the hot wall to drop, and therefore, heat and mass transfer decrease with the rise in Bingham number. The streamlines can prove the decrease in the convection process with the growth of the Bingham number as the inclined elliptical form of the core of the cavity alters to a circular shape. The figure corroborates the mentioned results of Bingham number effects evidently, where the maximum strength of streamline decreases with the augmentation of the Bingham number. It also depicts that the unyielded sections occupy more spaces in the cavity as the Bingham number augments. In fact, the rise in Bingham number causes the yielded regions to remove gradually.

Figure 7 depicts the local Nusselt and Sherwood numbers on the left wall in different Bingham numbers at $\mathrm{Ra}$ $=10^{5}, \mathrm{Da}=10^{-3}, \mathrm{~N}=0.1, \mathrm{Le}=2.5, \mathrm{Pr}=1$, and $\epsilon=0.4$. The local Nusselt number decreases with the enhancement of the Bingham number. In fact, for high values of the Bingham number, the viscous force more readily overcomes the buoyancy force, and as a result of this, no significant flow is induced within the enclosure. The local Sherwood number declines steadily as the Bingham number increases regularly. Table $\mathrm{V}$ indicates the average Nusselt and Sherwood numbers in different Bingham numbers at $\mathrm{Ra}=10^{5}, \mathrm{Da}=10^{-3}, \mathrm{~N}=0.1$, Le $=2.5$, and $\epsilon=0.4$. It depicts that the average Nusselt and Sherwood numbers drop gradually as the Bingham number rises.

\section{Darcy number effects}

The Darcy number is directly proportional to the permeability of the porous medium. Figure 8 shows the isotherms, streamlines, isoconcentrations, and yielded/unyielded parts in different Darcy numbers at $\mathrm{Bn}=1, \mathrm{Ra}=10^{5}, \mathrm{~N}=0.1$, Le $=2.5, \operatorname{Pr}=1$, and $\epsilon=0.4$. It exposes that the gradients of isotherms and isoconcentrations on the hot wall decline
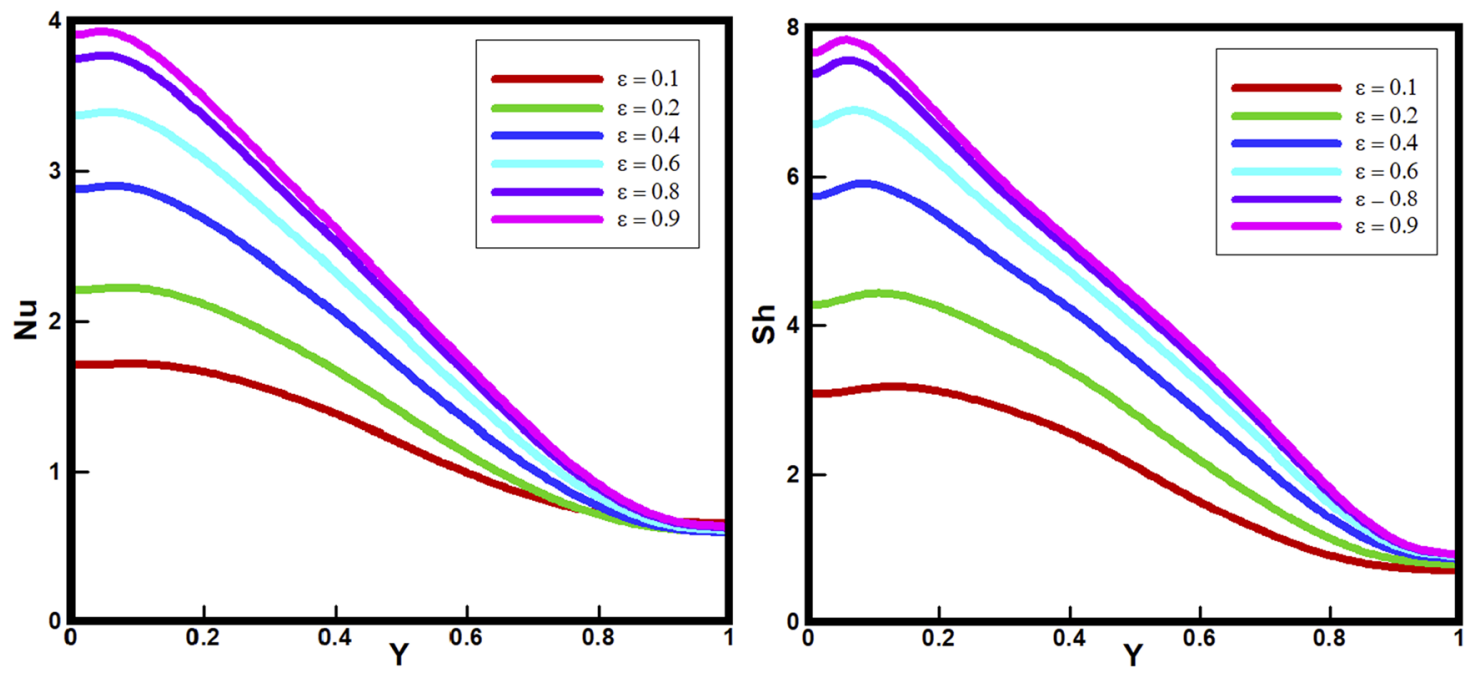

FIG. 11. The local Nusselt and Sherwood numbers on the hot wall in different porosities at $\mathrm{Da}=10^{-4}, \mathrm{Ra}=10^{5}, \mathrm{~N}=0.1, \mathrm{Bn}=1, \mathrm{Pr}=1$, and $\mathrm{Le}=2.5$. 
gradually as the Darcy number decreases from $\mathrm{Da}=10^{-2}$ to $10^{-6}$. The trend confirms that heat and mass transfer drop considerably due to the decline in the Darcy number. In addition, the streamline movement verifies the pattern as the elliptical shape of the streamline alters to circular ones, which demonstrates the convection process decrease substantially. The yielded/unyielded sections also change with the alteration of the Darcy number evidently. It shows that the yielded/unyielded zones alter slightly from $\mathrm{Da}=10^{-2}$ to
$10^{-3}$ although the concentration of the unyielded part rises marginally. At $\mathrm{Da}=10^{-4}$, the unyielded part changes completely in the shape and size compared to the Darcy numbers of $\mathrm{Da}=10^{-2}$ and $10^{-3}$, where two separate parallel sections are generated symmetrically. The two symmetric shape expands and becomes bigger in the center of the cavity at $\mathrm{Da}=10^{-5}$, compared to $\mathrm{Da}=10^{-4}$. At $\mathrm{Da}=10^{-6}$, the unyielded part fills nearly the entire of the cavity except some minor yielded sections close to the walls.
Isotherms
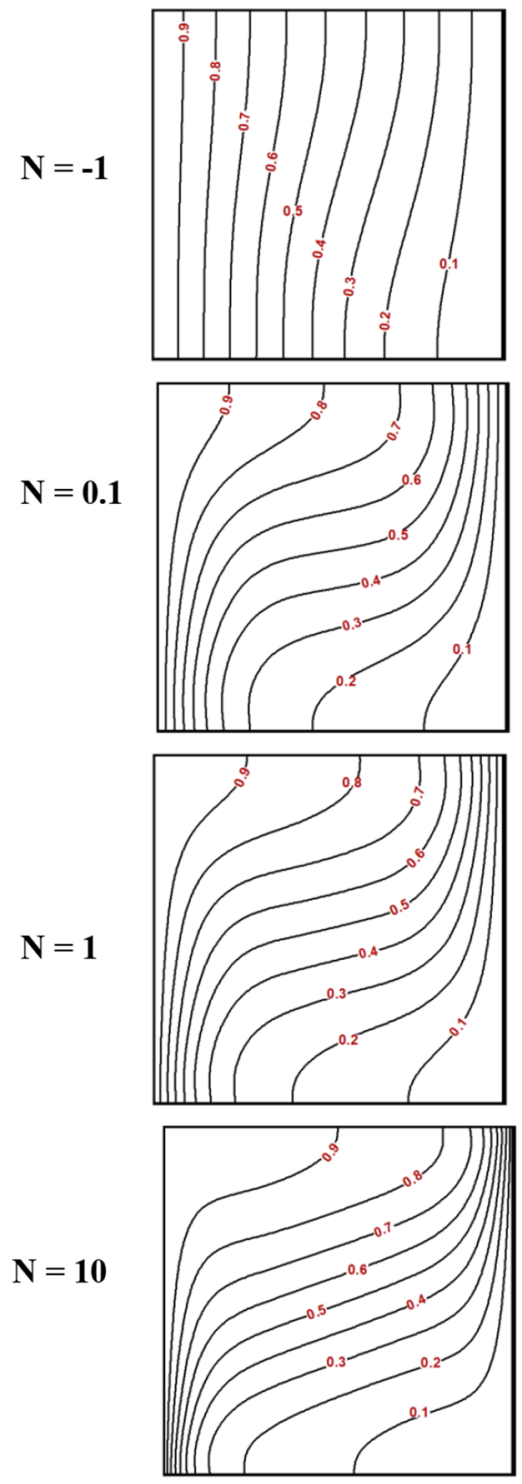

Streamlines
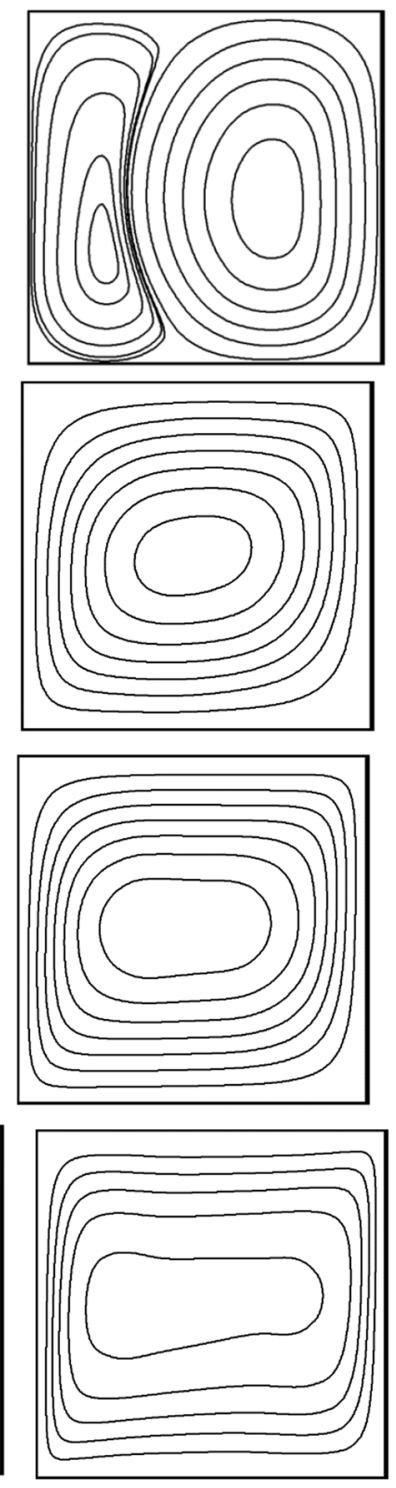

Isoconcentrations
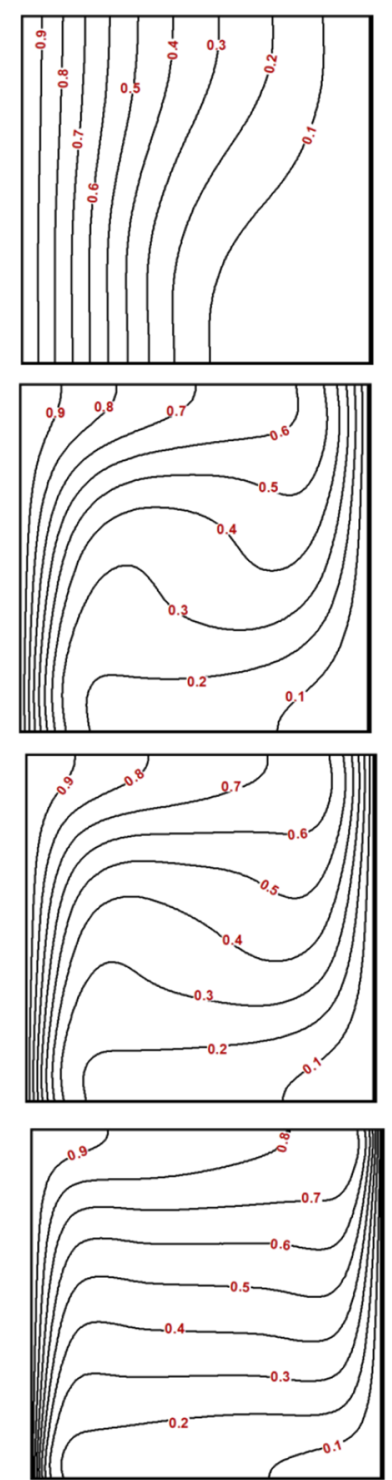

\section{Yielded/unyielded}
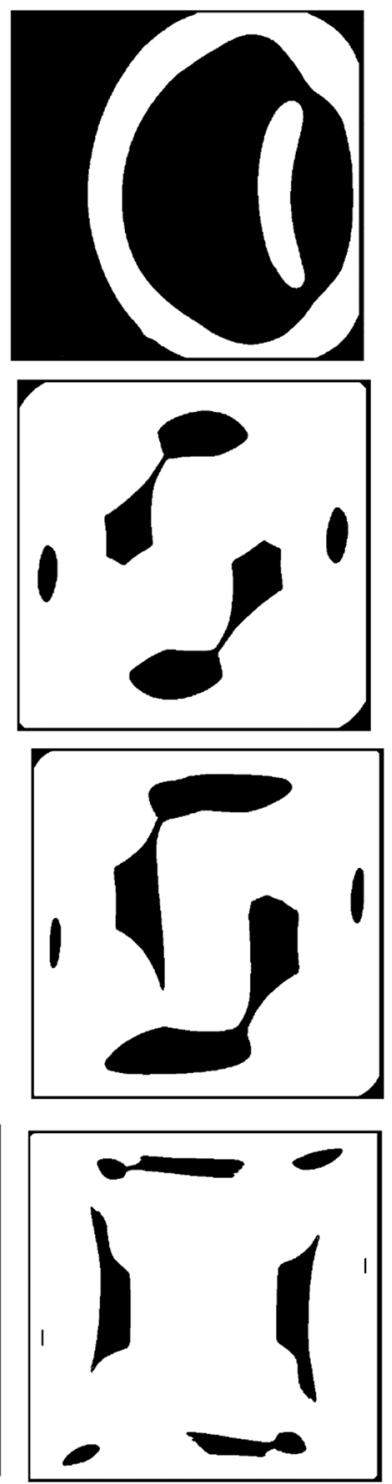

FIG. 12. The comparison of the isotherms, isoconcentrations, streamlines, and yielded/unyielded parts in different buoyancy ratio numbers at $\mathrm{Da}=10^{-3}, \mathrm{Ra}=10^{5}, \epsilon=0.4$, $\mathrm{Bn}=1, \mathrm{Pr}=1$, and $\mathrm{Le}=2.5$. 

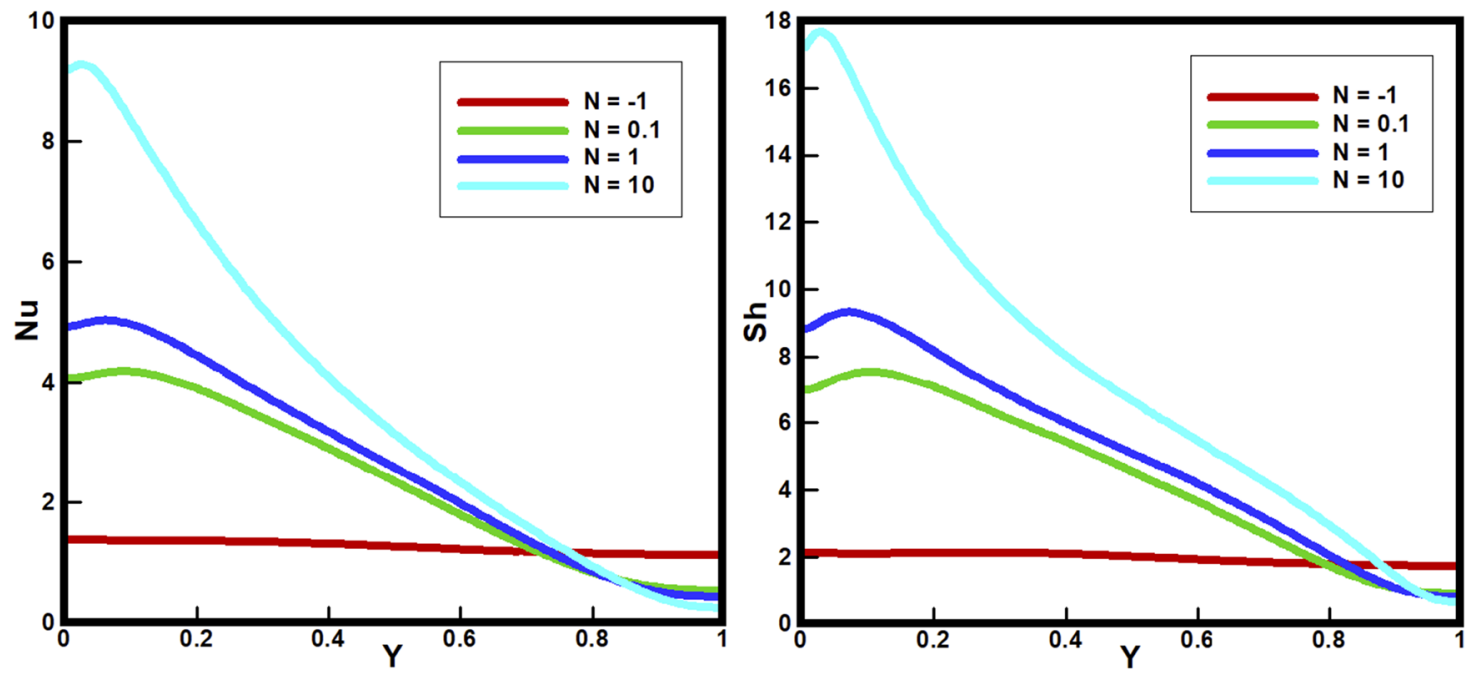

FIG. 13. The local Nusselt and Sherwood numbers on the hot wall in different buoyancy ratio numbers at $\mathrm{Da}=10^{-3}, \mathrm{Ra}=10^{5}, \epsilon=0.4, \mathrm{Bn}=1, \mathrm{Pr}=1$, and $\mathrm{Le}=2.5$.

Figure 9 shows the local Nusselt and Sherwood numbers on the hot wall for different Darcy numbers at $\mathrm{Ra}=10^{5}, \epsilon$ $=0.4, \mathrm{~N}=0.1, \mathrm{Bn}=1, \mathrm{Le}=2.5$, and $\mathrm{Pr}=1$. It shows that the local Nusselt and Sherwood numbers increase with the rise in the Darcy number gradually. Table VI shows the average Nusselt and Sherwood numbers in different Darcy numbers and porosities at $\mathrm{Bn}=1, \mathrm{Ra}=10^{5}, \mathrm{~N}=0.1$, Le $=2.5$, and $\operatorname{Pr}=1$. It depicts that the average Nusselt and Sherwood numbers in various porosities decrease due to the decline in the Darcy number from $\mathrm{Da}=10^{-2}$ to $10^{-6}$.

\section{Porosity effects}

Based on the materials with industrial importance, the porosity can vary between $\epsilon=0.1$ and $0.9,{ }^{25,26}$ so we have studied the parameter in the cited range. Figure 10 displays the isotherms, isoconcentrations, streamlines, and yielded/unyielded parts clearly for various porosities at $\mathrm{Da}=10^{-4}, \mathrm{Ra}=10^{5}, \mathrm{~N}=0.1, \mathrm{Bn}=1, \mathrm{Pr}=1$, and $\mathrm{Le}=2.5$. It illustrates that the gradient of the isotherms on the hot wall decreases as the porosity decreases. The trend has been confirmed by the streamlines as the core of them demonstrates that the convection process decreases with the drop in porosity. Furthermore, it exhibits that the unyielded section for certain Bingham and Rayleigh numbers increases with the drop in porosity. In fact, the increment of the unyielded sections at lower porosity causes the heat transfer to drop.

Figure 11 indicates the local Nusselt and Sherwood numbers for different porosities at $\mathrm{Da}=10^{-4}, \mathrm{Ra}=10^{5}, \mathrm{~N}=0.1$, $\mathrm{Bn}=1, \operatorname{Pr}=1$, and $\mathrm{Le}=2.5$. It is evident that the effect of porosity on the local Nusselt and Sherwood numbers is significant, where the increase in porosity enhances them steadily and therefore rises the convection process. Table VI illustrates that the average Nusselt and Sherwood numbers in various Darcy numbers rise substantially when the porosity increases.

\section{E. Buoyancy ratio effects}

Buoyancy ratio is the ratio between the solute and thermal buoyancy forces. It can be either positive or negative, and its sign depends on the coefficients of thermal and solutal expansions. The solutal and thermal buoyancy forces may be either augmenting or opposing each other. So, depending on the directions of the buoyancy forces, the problem can be either an aiding or opposing buoyancy condition.

TABLE VII. Comparison of the average Nusselt and Sherwood numbers for different buoyancy ratio numbers at $\epsilon=0.4, \mathrm{Bn}=1, \mathrm{Ra}=10^{5}, \mathrm{Le}=2.5$, and $\mathrm{Pr}=1$.

\begin{tabular}{lcccc}
\hline \hline & $\mathrm{N}=-1$ & $\mathrm{~N}=0.1$ & $\mathrm{~N}=1$ & $\mathrm{~N}=10$ \\
\hline $\mathrm{Da}=10^{-2}$ & & & \\
\hline $\mathrm{N} u_{\text {avg }}$ & 1.3328 & 2.7889 & 3.1782 & 4.7923 \\
$\mathrm{Sh}_{\text {avg }}$ & 2.0794 & 4.9336 & 5.7500 & 8.8990 \\
\hline $\mathrm{Da}=10^{-3}$ & & & \\
\hline $\mathrm{N} u_{\text {avg }}$ & 1.2573 & 2.3603 & 2.6456 & 3.7673 \\
$\mathrm{Sh}_{\text {avg }}$ & 1.9632 & 4.3985 & 5.0899 & 7.5334 \\
\hline $\mathrm{Da}=10^{-4}$ & & & & \\
\hline $\mathrm{N} u_{\text {avg }}$ & 1.2297 & 1.7190 & 1.8762 & 2.4797 \\
$\mathrm{Sh}_{\text {avg }}$ & 1.9039 & 3.4555 & 3.9174 & 5.4017 \\
\hline $\mathrm{Da}=10^{-5}$ & & & & \\
\hline $\mathrm{N} u_{\text {avg }}$ & 1.2244 & 1.3240 & 1.3923 & 1.6913 \\
$\mathrm{Sh}_{\text {avg }}$ & 1.8918 & 2.3006 & 2.5839 & 3.6448 \\
\hline $\mathrm{Da}=10^{-6}$ & & & & \\
\hline $\mathrm{Nu} u_{\text {avg }}$ & 1.2237 & 1.2354 & 1.2459 & 1.3069 \\
$\mathrm{Sh}_{\text {avg }}$ & 1.8906 & 1.9416 & 1.9904 & 2.2292 \\
\hline \hline
\end{tabular}


Figure 12 exhibits the isotherms, isoconcentrations, streamlines, and yielded/unyielded parts in different buoyancy ratios at $\mathrm{Da}=10^{-3}, \mathrm{Ra}=10^{5}, \epsilon=0.4, \mathrm{Bn}=1, \mathrm{Pr}=1$, and Le $=2.5$. The comparison between the isotherms demonstrates that the rise in buoyancy ratio from $\mathrm{N}=-1$ to 1 causes the gradient of the isotherms on the hot wall to increase significantly. Hence, the pattern clarifies that the augmentation of the buoyancy ratio enhances heat transfer. Moreover, the trend is observed in isoconcentrations as they incline to the hot wall and their gradient augments noticeably. As a result, mass transfer similar to heat transfer is improved by the increase in buoyancy ratio. The shapes of the streamlines in different buoyancy ratios can prove the cited result in the isotherms and isoconcentrations properly. At $\mathrm{N}=-1$, a secondary vortex close to the hot wall weakens the main vortex as it circulates in the opposite direction of the main vortex counterclockwise. In addition, it is evident that the main circulation in the cavity move toward the hot wall as the buoyancy ratio enhances from $\mathrm{N}=0.1$ to 1 , and moreover, the separated vortexes in the core of the cavity become uniform with an apparent inclination to the hot wall.
It is clear that yielded/unyielded sections alter as the buoyancy ratio changes. At $\mathrm{N}=-1$, an unyielded zone is formed close to the hot wall and two small parts are generated in the center of the cavity. But, in the positive buoyancy ratio, the unyielded section is created in the center of the cavity; however, the size of the unyielded part drops as the buoyancy ratio increases from $\mathrm{N}=0.1$ to 10 . In addition, it demonstrates that the change of the buoyancy ratio declines the symmetric shape of the yielded/unyielded zones, especially for negative buoyancy ratios.

Figure 13 displays the local Nusselt and Sherwood numbers on the hot wall for different buoyancy ratio numbers at $\mathrm{Da}=10^{-3}, \mathrm{Ra}=10^{5}, \epsilon=0.4, \mathrm{Bn}=1, \mathrm{Pr}=1$, and Le $=2.5$. It shows that the local Nusselt and Sherwood numbers increase with the rise in buoyancy ratio although the rise is more significant from $\mathrm{N}=-1$ to 0.1 compared to the increase from $\mathrm{N}=0.1$ to 1 . Table VII reveals the average Nusselt and Sherwood numbers in different buoyancy ratio numbers for various Darcy numbers at $\mathrm{Ra}=10^{5}, \epsilon=0.4, \mathrm{Bn}=1, \mathrm{Pr}=1$, and Le $=2.5$. It demonstrates that the average Nusselt and Sherwood numbers in

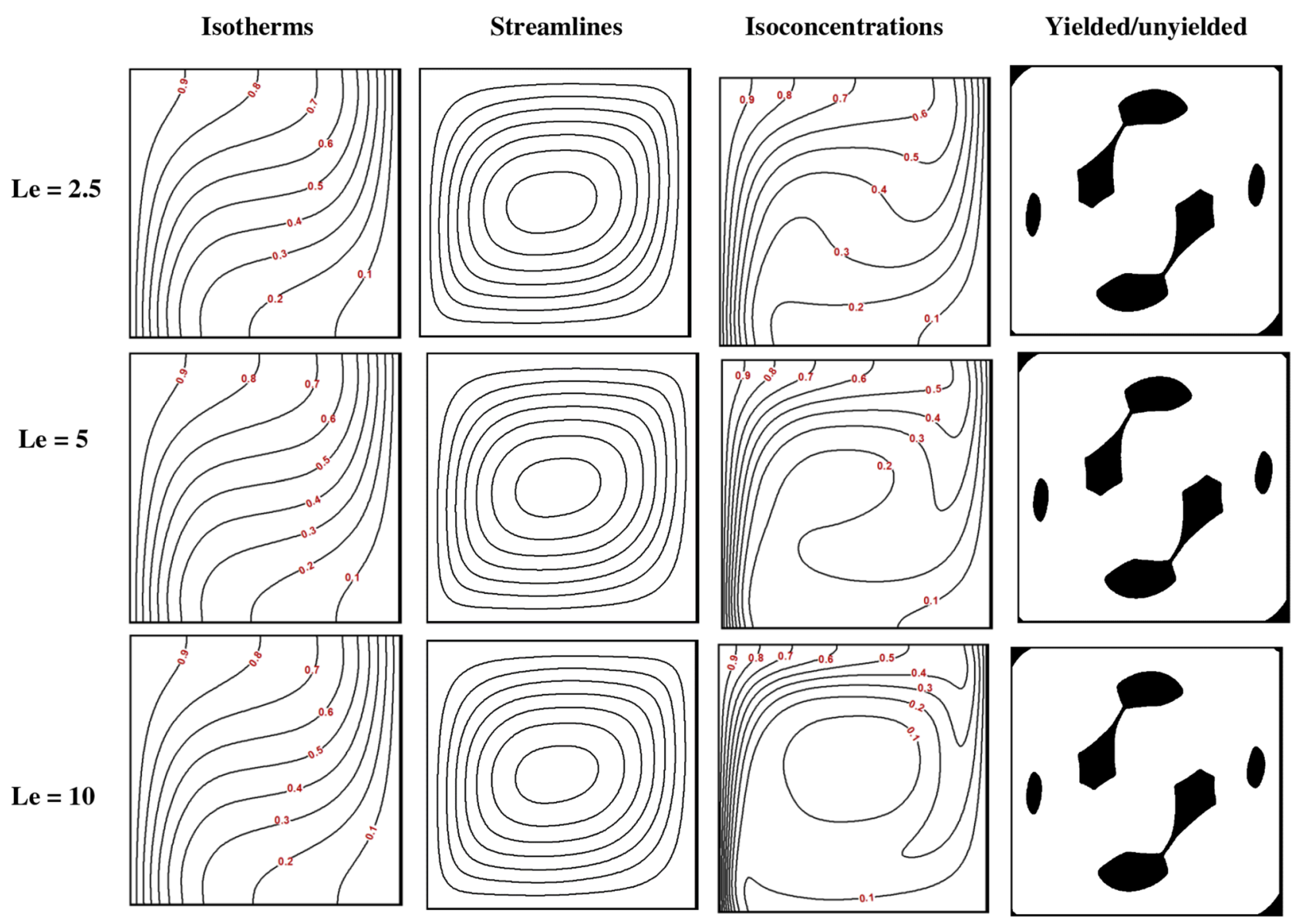

FIG. 14. The comparison of the isotherms, isoconcentrations, streamlines, and yielded/unyielded parts in different Lewis numbers at $\mathrm{Da}=10^{-3}, \mathrm{Ra}=10^{5}, \epsilon=0.4, \mathrm{Bn}=1$, $\operatorname{Pr}=1$, and $\mathrm{N}=0.1$. 

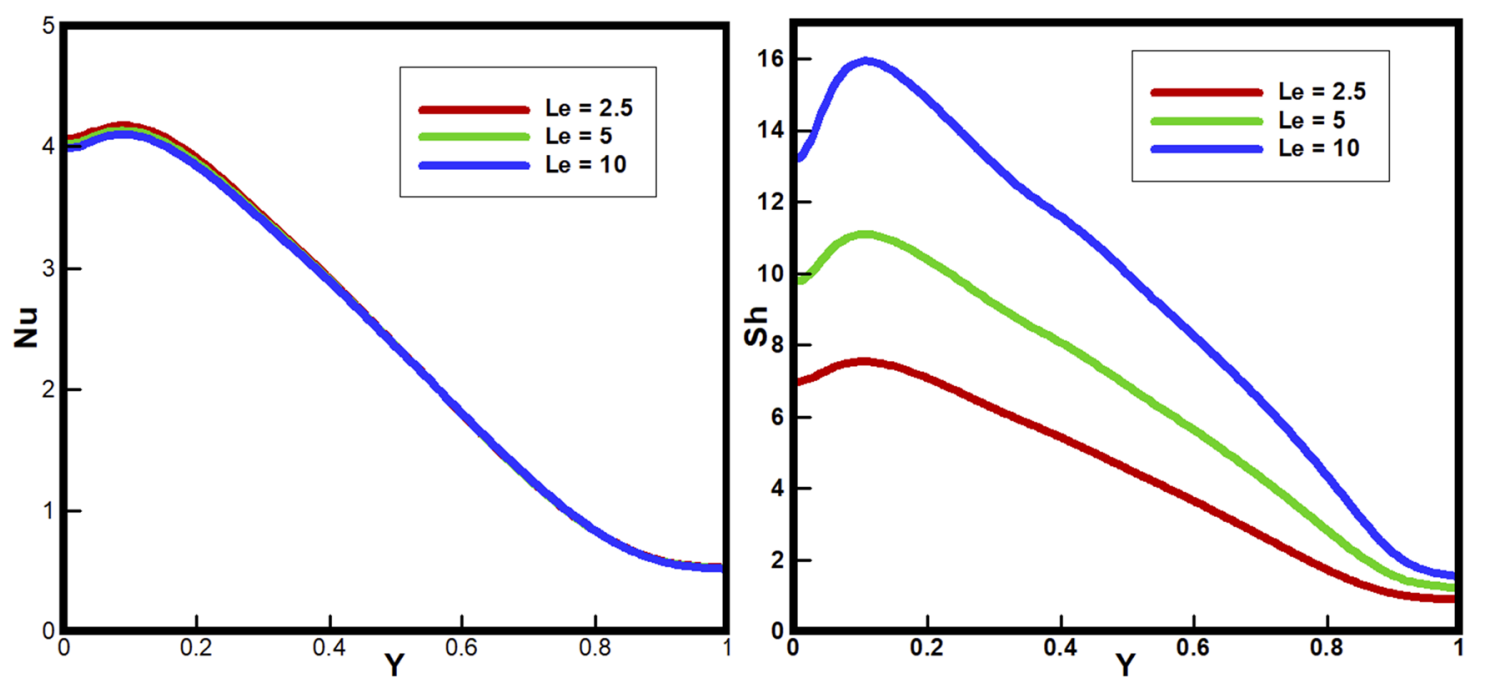

FIG. 15. The local Nusselt and Sherwood numbers on the hot wall in different Lewis numbers at $\mathrm{Da}=10^{-3}, \mathrm{Ra}=10^{5}, \epsilon=0.4, \mathrm{Bn}=1$, $\operatorname{Pr}=1$, and $\mathrm{N}=0.1$.

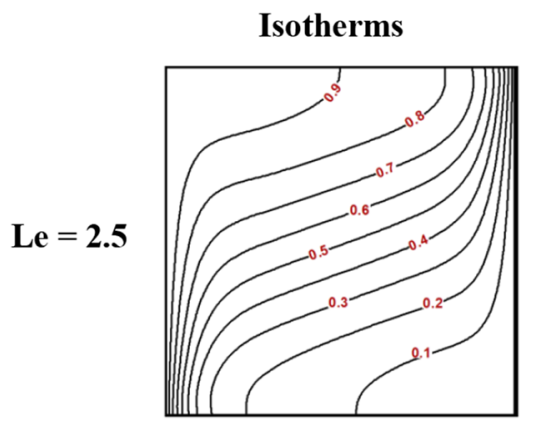

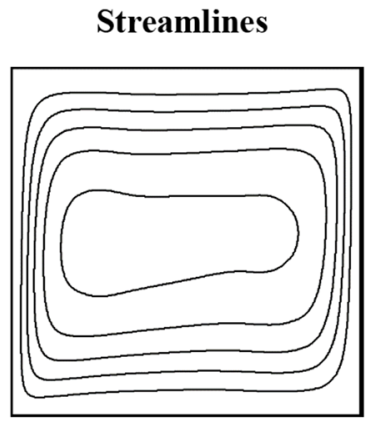

Isoconcentrations
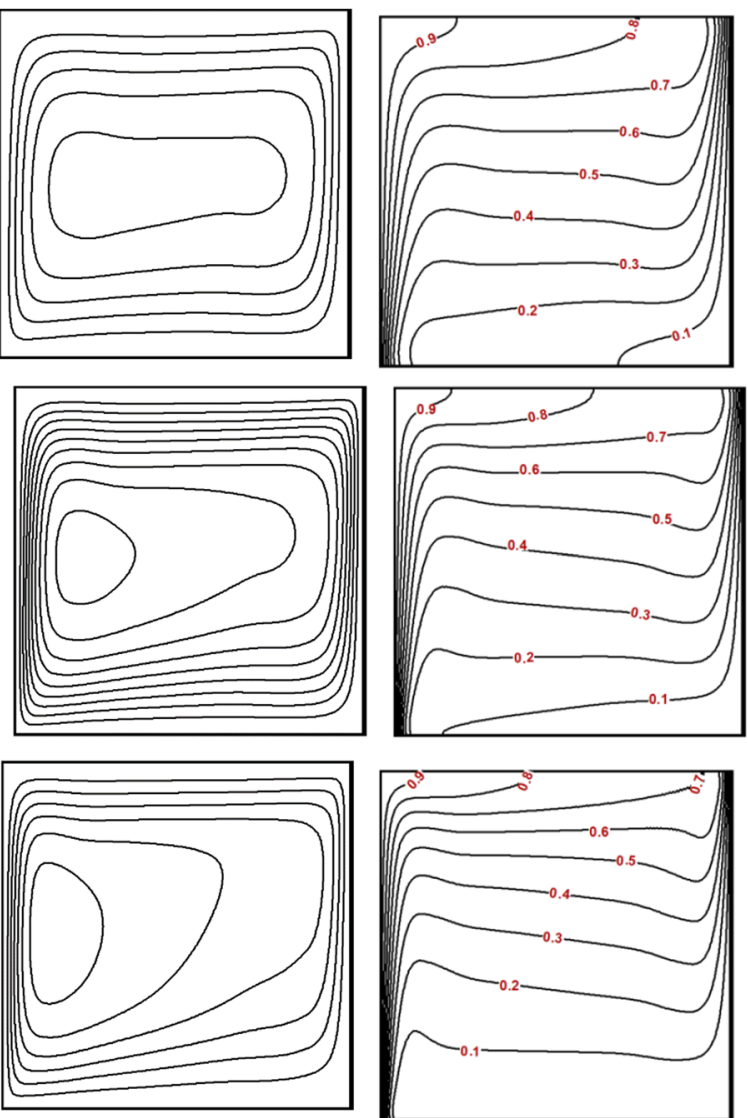

Yielded/unyielded
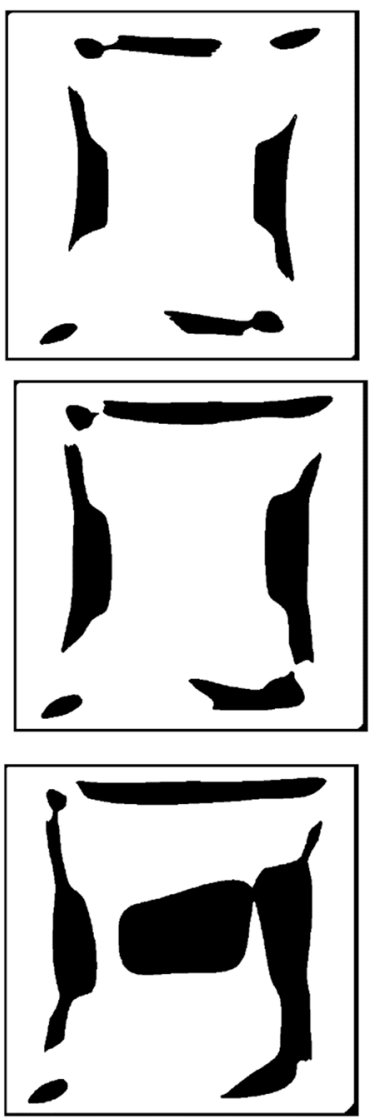

FIG. 16. The comparison of the isotherms, isoconcentrations, streamlines, and yielded/unyielded parts in different Lewis numbers at $\mathrm{Da}=10^{-3}, \mathrm{Ra}=10^{5}, \epsilon=0.4, \mathrm{Bn}=1, \mathrm{Pr}$ $=1$, and $\mathrm{N}=10$. 
various Darcy numbers rise as the buoyancy ratio increases from $\mathrm{N}=-1$ to 10 .

\section{F. Lewis number effects}

The Lewis number deals with the relative influence of thermal and mass diffusions. It has an effect on the thicknesses of the thermal and solutal boundary layers. When the Lewis number increases, the solutal boundary layer becomes thinner and the Sherwood number enhances. Figure 14 depicts the isotherms, isoconcentrations, streamlines, and yielded/unyielded parts in different Lewis numbers at $\mathrm{Da}=10^{-3}, \mathrm{Ra}=10^{5}, \epsilon=0.4, \mathrm{Bn}=1, \mathrm{Pr}=1$, and $\mathrm{N}=0.1$. The contours exhibit that the density of the isoconcentrations on the hot wall grows with the increase in Lewis numbers. The pattern confirms that mass transfer enhances with the rise in Lewis number generally without consideration to the Bingham number. However, for the studied range of Lewis numbers, the isotherms, streamlines, and yielded/unyielded sections do not alter significantly. The trend can be proved by Fig. 15, where the local Nusselt number is nearly identical for different Lewis numbers except in the maximum part where it changes slightly, but the local Sherwood number rises considerably as the Lewis number enhances. In fact, the main reason for the minor effect of the Lewis number on isotherms, streamlines, and yielded/unyielded sections is the small value of the buoyancy ratio $(\mathrm{N}=0.1)$, which causes the concentration effect on the momentum equation to be minor. To prove the statement, the buoyancy ratio of $\mathrm{N}=10$ has been studied for different Lewis numbers in Fig. 16. It is clear that not only isoconcentrations have changed, but the isotherms, streamlines, and yielded/unyielded sections have also been affected considerably by the increase in Lewis number. Table VIII indicates the average Nusselt and Sherwood numbers in different Lewis numbers for various Darcy numbers at $\mathrm{Ra}=10^{5}, \epsilon=0.4, \mathrm{Bn}=1$, $\operatorname{Pr}=1$, and $\mathrm{N}=0.1$ and 10. It demonstrates that the average Sherwood number enhances considerably at $\mathrm{N}=0.1$ and 10 as the Lewis number rises. However, due to the increase in the Lewis number, the average Nusselt number declines slightly at $\mathrm{N}=0.1$ and drops significantly at $\mathrm{N}=10$.

\section{G. Prandtl number effects}

The Prandtl number is the ratio of the momentum diffusivity to the thermal diffusivity of the fluid. The Prandtl number can be taken to represent the ratios of hydrodynamic boundary layer to thermal boundary layer thicknesses. Figure 17 exhibits the isotherms, isoconcentrations, streamlines, and yielded/unyielded parts in different Prandtl numbers at $\mathrm{Da}=10^{-5}, \mathrm{Ra}=10^{5}, \epsilon=0.1, \mathrm{Bn}=0.1, \mathrm{Le}=2.5$, and $\mathrm{N}$ $=0.1$. It is clear that the effect of the Prandtl number on the isotherms, isoconcentrations, and streamlines is marginal. In addition, it exhibits that the unyielded zone expands gradually when the Prandtl number increases from $\mathrm{Pr}=1$ to 100. Table IX shows the average Nusselt and Sherwood numbers in different Prandtl numbers at $\mathrm{Ra}=10^{5}, \epsilon=0.1, \mathrm{Bn}=0.1$, Le $=2.5$, and $\mathrm{N}=0.1$. It illustrates that the heat and mass transfer enhance at $\mathrm{Da}=10^{-3}$ as Prandtl number rises in the small value of the Bingham number while the rise is marginal at $\mathrm{Da}=10^{-5}$. A large
TABLE VIII. Comparison of the average Nusselt and Sherwood numbers for different Lewis numbers at $\epsilon=0.4, \mathrm{Bn}=1, \mathrm{Ra}=10^{5}$, and $\mathrm{Pr}=1$.

\begin{tabular}{|c|c|c|c|}
\hline & $\mathrm{Le}=2.5$ & $\mathrm{Le}=5$ & $\mathrm{Le}=10$ \\
\hline \multicolumn{4}{|c|}{$\mathrm{Da}=10^{-2}$} \\
\hline \multicolumn{4}{|c|}{$N=0.1$} \\
\hline $\mathrm{Nu} u_{\text {avg }}$ & 2.7889 & 2.7683 & 2.7574 \\
\hline $\begin{array}{l}S h_{\text {avg }} \\
N=10\end{array}$ & 4.9336 & 7.1881 & 10.0929 \\
\hline $\mathrm{N} u_{\text {avg }}$ & 4.7923 & 3.4237 & 2.1635 \\
\hline$S h_{\text {avg }}$ & 8.8989 & 12.3191 & 17.6825 \\
\hline \multicolumn{4}{|c|}{$\mathrm{Da}=10^{-3}$} \\
\hline \multicolumn{4}{|l|}{$\mathrm{N}=0.1$} \\
\hline$N u_{\text {avg }}$ & 2.3603 & 2.3455 & 2.3361 \\
\hline $\begin{array}{l}S h_{\text {avg }} \\
N=10\end{array}$ & 4.3985 & 6.5505 & 9.4189 \\
\hline $\mathrm{Nu} u_{\text {avg }}$ & 3.7673 & 2.7477 & 1.9106 \\
\hline$S h_{\text {avg }}$ & 7.5334 & 11.1366 & 16.4706 \\
\hline \multicolumn{4}{|c|}{$\mathrm{Da}=10^{-4}$} \\
\hline \multicolumn{4}{|c|}{$\mathrm{N}=0.1$} \\
\hline$N u_{\text {avg }}$ & 1.7190 & 1.7116 & 1.7087 \\
\hline $\begin{array}{l}S h_{\text {avg }} \\
\mathrm{N}=10\end{array}$ & 3.4555 & 5.2847 & 7.3958 \\
\hline$N u_{\text {avg }}$ & 2.4797 & 1.9709 & 1.6208 \\
\hline$S h_{\text {avg }}$ & 5.4017 & 8.5130 & 12.7494 \\
\hline \multicolumn{4}{|c|}{$\mathrm{Da}=10^{-5}$} \\
\hline \multicolumn{4}{|c|}{$\mathrm{N}=0.1$} \\
\hline $\mathrm{N} u_{\text {avg }}$ & 1.3240 & 1.3228 & 1.3213 \\
\hline $\begin{array}{l}S h_{\text {avg }} \\
\mathrm{N}=10\end{array}$ & 2.3006 & 3.4459 & 4.9277 \\
\hline$N u_{\text {avg }}$ & 1.6913 & 1.5580 & 1.4722 \\
\hline$S h_{\text {avg }}$ & 3.6448 & 5.7791 & 8.4702 \\
\hline \multicolumn{4}{|c|}{$\mathrm{Da}=10^{-6}$} \\
\hline \multicolumn{4}{|c|}{$\mathrm{N}=0.1$} \\
\hline$N u_{\text {avg }}$ & 1.2354 & 1.2352 & 1.2351 \\
\hline $\begin{array}{l}S h_{\text {avg }} \\
N=10\end{array}$ & 1.9416 & 2.7767 & 3.9440 \\
\hline $\mathrm{N} u_{\text {avg }}$ & 1.3011 & 1.2905 & 1.2722 \\
\hline$S h_{\text {avg }}$ & 2.2146 & 3.3164 & 4.8462 \\
\hline
\end{tabular}

value of the Prandtl number corresponds to a thin boundary layer, and hence, the Nusselt number should increase with the Prandtl number, for Newtonian fluids and small Bingham numbers. For high Bingham numbers, the effects of the buoyancy force become increasingly weak in comparison with the viscous effects with increasing Prandtl number when other parameters are held constant. So, the average Nusselt and Sherwood numbers drop at the high values of the Bingham number. But, this effect is relatively weak for small values of Bingham numbers (e.g., $\mathrm{Bn}=0.1$ ), where an increase in Prandtl number acts to reduce the thermal boundary layer thickness which in turn acts to increase the heat transfer rate. 

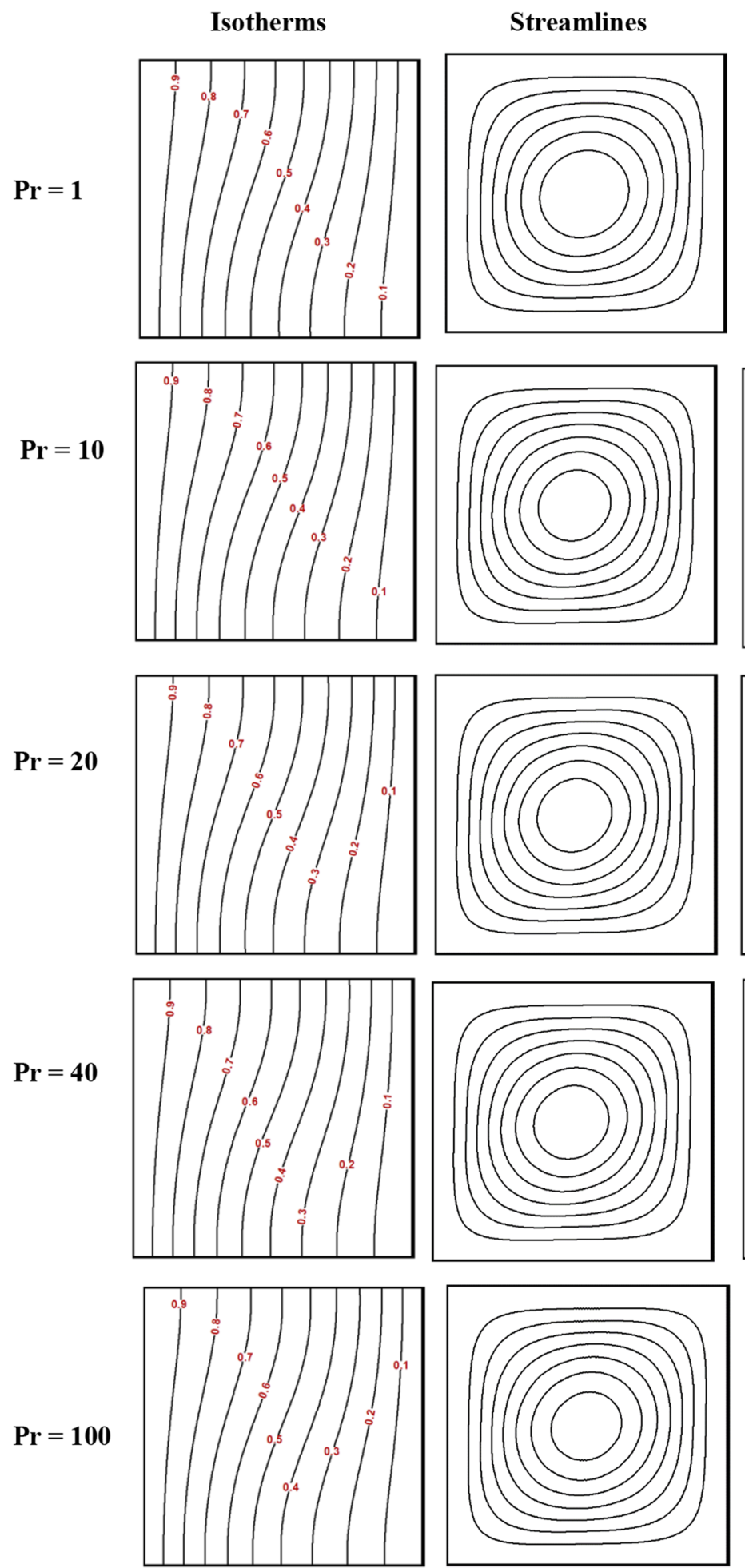

Isoconcentrations
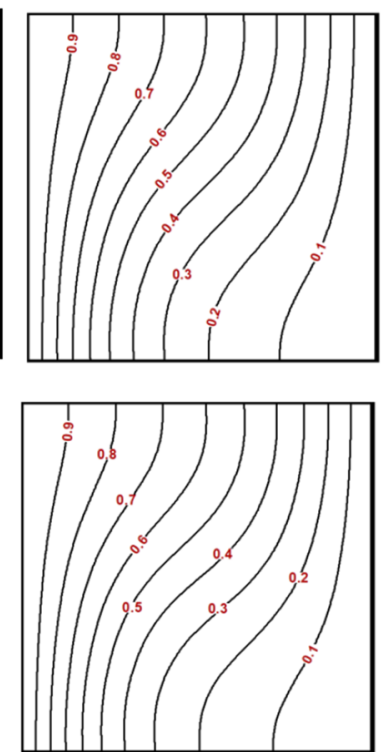

Yielded/unyielded
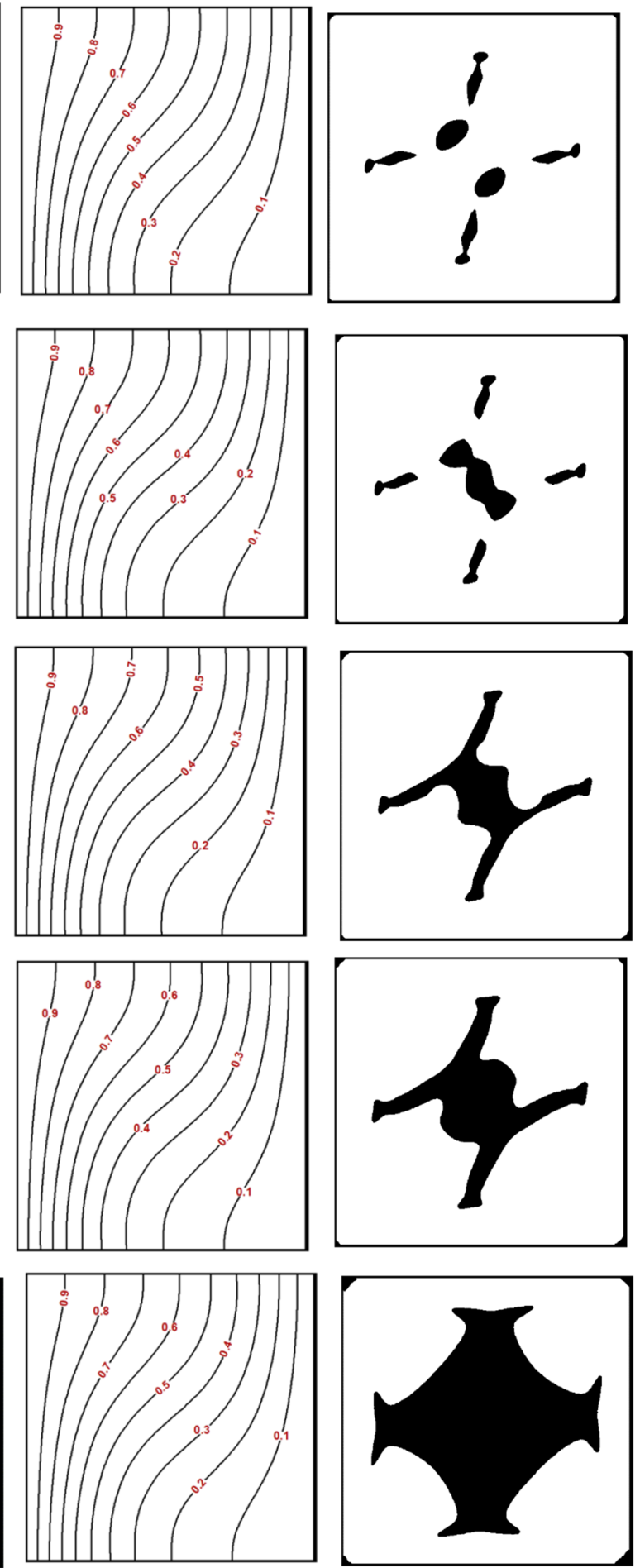

FIG. 17. The comparison of the isotherms, streamlines, isoconcentrations, and yielded/unyielded parts in different Prandtl numbers at $\mathrm{Da}=10^{-5}, \mathrm{Ra}=10^{5}, \epsilon=0.1, \mathrm{Bn}=0.1$, $\mathrm{Le}=2.5$, and $\mathrm{N}=0.1$. 
TABLE IX. Comparison of the average Nusselt and Sherwood numbers for different Prandtl numbers at $\epsilon=0.1, \mathrm{Bn}=0.1, \mathrm{Ra}=10^{5}, \mathrm{~N}=0.1$, and $\mathrm{Le}=2.5$.

\begin{tabular}{lccccc}
\hline \hline & $\operatorname{Pr}=1$ & $\operatorname{Pr}=10$ & $\operatorname{Pr}=20$ & $\operatorname{Pr}=40$ & $\operatorname{Pr}=100$ \\
\hline $\mathrm{Da}=10^{-3}$ & & & & & \\
\hline $\mathrm{Nu}_{\text {avg }}$ & 1.5889 & 1.8810 & 1.9088 & 1.9234 & 1.9328 \\
$\mathrm{Sh}_{\text {avg }}$ & 2.8381 & 3.1871 & 3.2122 & 3.2253 & 3.2363 \\
\hline $\mathrm{Da}=10^{-5}$ & & & & & \\
\hline $\mathrm{Nu}_{\text {avg }}$ & 1.0783 & 1.0871 & 1.0878 & 1.0882 & 1.0847 \\
$\mathrm{Sh}_{\text {avg }}$ & 1.5993 & 1.6383 & 1.6412 & 1.6435 & 1.6311 \\
\hline \hline
\end{tabular}

\section{CONCLUDING REMARKS}

Double diffusive natural convection of viscoplastic fluid in a porous cavity has been analyzed by LBM. This study has been conducted for the pertinent parameters in the following ranges: the Rayleigh number $\left(\mathrm{Ra}=10^{4}-10^{6}\right)$, Darcy number (Da $=10^{-2}$ to $\left.10^{-6}\right)$, Prandtl number $(\operatorname{Pr}=1-100)$, porosity $(\epsilon=0.1-$ $0.9)$, Lewis number $(\mathrm{Le}=2.5,5$, and 10$)$, and buoyancy ratio $(\mathrm{N}$ $=-1,0.1,1$, and 10). The Bingham number is also studied in a wide range. The main conclusions of the present investigation can be summarized as follows:

- The increase in Lewis number augments the mass transfer in various Bingham numbers in high and low buoyancy ratios of $\mathrm{N}=0.1$ and 10 . But, the heat transfer decreases slightly at $\mathrm{N}=0.1$ and declines significantly at $\mathrm{N}=10$.

- The increase in Lewis number from Le $=2.5$ to 10 does not affect the unyielded zones noticeably at $\mathrm{N}=0.1$, but the unyielded sections are expanded by the rise in Lewis number at $\mathrm{N}=10$.

- The drop in the Darcy number from $\mathrm{Da}=10^{-2}$ to $10^{-6}$ weakens the convection process of heat and mass transfer and alters the shape and size of the unyielded sections.

- The increases in porosity in a specific Darcy number causes heat and mass transfer to rise, while the unyielded section diminishes gradually.

- The enhancement of the buoyancy ratio increases heat and mass transfer considerably. However, the increase is not uniform in different Bingham numbers and porosities.

- The increase in the buoyancy ratio from $\mathrm{N}=0.1$ to 1 expands the unyielded zone slightly, while the rise in the buoyancy ratio from $\mathrm{N}=1$ to 10 diminishes the unyielded zone marginally. But, the change in the buoyancy ratio from $\mathrm{N}=0.1$ to -1 develops the unyielded zones considerably.

- It is observed that the negative buoyancy ratio removes the symmetric shape of the unyielded zone.

- The increase in Prandtl number enlarges the unyielded section in different Darcy numbers.

- In the small Bingham number of $\mathrm{Bn}=0.1$, the rise in Prandtl number from $\mathrm{Pr}=1$ to 100 enhances heat and mass transfer by nearly $20 \%$ in higher Darcy number
$\left(\mathrm{Da}=10^{-3}\right)$, but it has a minor influence on heat and mass transfer (approximately by $0.5 \%$ ) at lower Darcy numbers $\left(\mathrm{Da}=10^{-5}\right)$.

\section{NOMENCLATURE}

$\mathbf{A}_{1}$ the first Rivlin-Ericksen tensor

Bn Bingham number

C concentration

c lattice speed

$c_{p} \quad$ specific heat capacity at constant pressure

D mass diffusivity

Da Darcy number

F external forces

$f_{\alpha} \quad$ density distribution functions for the specific node of $\alpha$

$f_{\alpha}^{e q}$ equilibrium density distribution functions for the specific node of $\alpha$

$g_{\alpha} \quad$ internal energy distribution functions for the specific node of $\alpha$

$g_{\alpha}^{e q}$ equilibrium internal energy distribution functions for the specific node of $\alpha$

$g$ gravitational acceleration

$h_{\alpha}$ internal concentration distribution functions for the specific node of $\alpha$

$h_{\alpha}^{e q}$ equilibrium internal concentration distribution functions for the specific node of $\alpha$

$k$ thermal conductivity

$\mathrm{K}$ permeability of the porous medium

L length of the cavity

Le Lewis number

$\mathrm{N}$ buoyancy ratio

$\mathrm{Nu}$ Nusselt number

$p$ pressure

Pr Prandtl number

Ra Rayleigh number

Sh Sherwood number

$\mathrm{T}$ temperature

$t$ time

$x, y \quad$ Cartesian coordinates

$u$ the volume averaged velocity in $\mathrm{x}$ direction

$\mathrm{U}$ the buoyancy velocity scale

$v$ the volume averaged velocity in y direction

\section{Greek letters}

$\beta_{\mathrm{T}} \quad$ thermal expansion coefficient

$\beta_{\mathrm{C}} \quad$ solutal expansion coefficient

$\phi \quad$ relaxation time

$\tau$ shear stress

$\tau$ yield stress

$\xi \quad$ discrete particle speeds

$\Delta x \quad$ lattice spacing in $x$ direction

$\Delta y \quad$ lattice spacing in $y$ direction

$\Delta t \quad$ time increment

$\alpha \quad$ thermal diffusivity

$\rho$ density of fluid

$\eta \quad$ dynamic viscosity

$\eta_{p} \quad$ plastic viscosity

$\epsilon \quad$ porosity of the porous medium 


\section{Subscripts}

avg average

$C$ cold

e effective

$\mathrm{H}$ hot

L left

$\mathrm{R}$ right

$x, y \quad$ Cartesian coordinates

$\alpha \quad$ specific node

$m$ modified

\section{REFERENCES}

${ }^{1}$ A. A. Mohamad and A. Kuzmin, "A critical evaluation of force term in lattice Boltzmann method, natural convection problem," Int. J. Heat Mass Transfer 53, 990 (2010).

${ }^{2}$ A. A. Mehrizi, M. Farhadi, and S. Shayamehr, "Natural convection flow of $\mathrm{Cu}$-Water nanofluid in horizontal cylindrical annuli with inner triangular cylinder using lattice Boltzmann method," Int. Commun. Heat Mass Transfer 44, 147-156 (2013).

${ }^{3}$ H. R. Ashorynejad, A. A. Mohamad, and M. Sheikholeslami, "Magnetic field effects on natural convection flow of a nanofluid in a horizontal cylindrical annulus using lattice Boltzmann method," Int. J. Therm. Sci. 64, 240 (2013).

${ }^{4}$ H. Sajjadi, M. Salmanzadeh, G. Ahmadi, and S. Jafari, "Turbulent indoor airflow simulation using hybrid LES/RANS model utilizing lattice Boltzmann method," Comput. Fluids 150, 66 (2017).

${ }^{5}$ H. Sajjadi, M. Salmanzadeh, G. Ahmadi, and S. Jafari, "Simulations of indoor airflow and particle dispersion and deposition by the lattice Boltzmann method using LES and RANS approaches," Build. Environ. 102, 1 (2016).

${ }^{6}$ H. Sajjadi, A. A. Delouei, M. Atashafrooz, and M. Sheikholeslami, "Double MRT lattice Boltzmann simulation of 3-D MHD natural convection in a cubic cavity with sinusoidal temperature distribution utilizing nanofluid," Int. J. Heat Mass Transfer 126, 489 (2018).

${ }^{7}$ H. Sajjadi, A. A. Delouei, M. Sheikholeslami, M. Atashafrooz, and S. Succi, "Simulation of three dimensional MHD natural convection using double MRT lattice Boltzmann method," Physica A 515, 474 (2019).

${ }^{8}$ H. Sajjadi, M. Salmanzadeh, G. Ahmadi, and S. Jafari, "Lattice Boltzmann method and RANS approach for simulation of turbulent flows and particle transport and deposition," Particuology 30, 62 (2017).

${ }^{9}$ H. Sajjadi and GH. R. Kefayati, "Turbulent and laminar natural convection in a square cavity utilizing lattice Boltzmann method," Heat Transfer-Asian Res. 45, 795 (2016).

${ }^{10} \mathrm{~K}$. Javadi and K. Kazemi, "Microgravity modulation effects on free convection problems LBM simulation," Phys. Fluids 30, 017104 (2018).

${ }^{11}$ Z. Chen, C. Shu, and D. Tan, "Three-dimensional simplified and unconditionally stable lattice Boltzmann method for incompressible isothermal and thermal flows," Phys. Fluids 29, 053601 (2017).

${ }^{12}$ Y. Zhang and Y. Cao, "A numerical study on the non-Boussinesq effect in the natural convection in horizontal annulus," Phys. Fluids 30, 040902 (2018).

${ }^{13}$ R. Mohebbi, M. Izadi, and A. J. Chamkha, "Heat source location and natural convection in a C-shaped enclosure saturated by a nanofluid," Phys. Fluids 29, 122009 (2017).

${ }^{14} \mathrm{~K}$. Walayat, Z. Zhang, K. Usman, J. Chang, and M. Liu, "Dynamics of elliptic particle sedimentation with thermal convection," Phys. Fluids 30, 103301 (2018).

${ }^{15} \mathrm{M}$. Sadr and M. H. Gorji, "A continuous stochastic model for nonequilibrium dense gases," Phys. Fluids 29, 122007 (2017).

${ }^{16}$ R. R. Huilgol and GH. R. Kefayati, "From mesoscopic models to continuum mechanics: Newtonian and non-Newtonian fluids," J. Non-Newtonian Fluid Mech. 233, 146 (2016).
${ }^{17}$ R. R. Huilgol and GH. R. Kefayati, "A particle distribution function approach to the equations of continuum mechanics in Cartesian, cylindrical and spherical coordinates: Newtonian and non-Newtonian fluids," J. Non-Newtonian Fluid Mech. 251, 119 (2018).

${ }^{18} \mathrm{GH}$. R. Kefayati, H. Tang, A. Chan, and X. Wang, "A lattice Boltzmann model for thermal non-Newtonian fluid flows through porous media," Comput. Fluids 176, 226 (2018).

${ }^{19}$ D. A. Nield and A. Bejan, Convection in Porous Media, 3rd ed. (Springer, 2006), Vol. XXIV.

${ }^{20}$ K. Vafai, Handbook of Porous Media (Marcel Dekker, New York, 2005).

${ }^{21}$ P. Nithiarasu, K. N. Seetharamu, and T. Sundararajan, "Natural convection heat transfer in a fluid saturated variable porosity medium," Int. J. Heat Mass Transfer 40, 3955 (1997)

${ }^{22} \mathrm{Z}$. Guo and T. S. Zhao, "A lattice Boltzmann model for convection heat transfer in porous media," Numer. Heat Transfer, Part B 47, 157 (2005).

${ }^{23}$ Q. Liu, Y. L. He, Q. Li, and W. Q. Tao, "A multiple-relaxation-time lattice Boltzmann model for convection heat transfer in porous media," Int. J. Heat Mass Transfer 73, 761 (2014).

${ }^{24} \mathrm{~L}$. Wang, J. Mi, and Z. Guo, "A modified lattice Bhatnagar-Gross-Krook model for convection heat transfer in porous media," Int. J. Heat Mass Transfer 94, 269 (2016).

${ }^{25}$ D. Das, P. Biswal, M. Roy, and T. Basak, "Role of the importance of 'Forchheimer term' for visualization of natural convection in porous enclosures of various shapes," Int. J. Heat Mass Transfer 97, 1044 (2016).

${ }^{26} \mathrm{D}$. Das and T. Basak, "Role of discrete heating on the efficient thermal management within porous square and triangular enclosures via heatline approach," Int. J. Heat Mass Transfer 112, 489 (2017).

${ }^{27}$ K. M. Khanafer and A. J. Chamkha, "Mixed convection flow in a lid-driven enclosure filled with a fluid-saturated porous medium," Int. J. Heat Mass Transfer 42, 2465 (1999).

${ }^{28}$ I. Aleshkova and M. Sheremet, "Unsteady conjugate natural convection in a square enclosure filled with a porous medium," Int. J. Heat Mass Transfer 53, 5308 (2010).

${ }^{29} \mathrm{M}$. Sheremet and I. Pop, "Conjugate natural convection in a square porous cavity filled by a nanofluid using Buongiorno's mathematical model," Int. J. Heat Mass Transfer 79, 137 (2014).

${ }^{30} \mathrm{M}$. Sheremet, I. Pop, and M. Rahman, "Three-dimensional natural convection in a porous enclosure filled with a nanofluid using Buongiorno's mathematical model," Int. J. Heat Mass Transfer 82, 396 (2015).

${ }^{31}$ H. Oztop, K. Al-Salem, Y. Varol, and I. Pop, "Natural convection heat transfer in a partially opened cavity filled with porous media," Int. J. Heat Mass Transfer 54, 2253 (2011).

${ }^{32} \mathrm{O}$. V. Trevisan and A. Bejan, "Mass and heat transfer by natural convection in a vertical slot filled with porous medium," Int. J. Heat Mass Transfer 29, 403 (1986).

${ }^{33}$ B. Goyeau, J. P. Songbe, and D. Gobin, "Numerical study of doublediffusive natural convection in a porous cavity using the Darcy-Brinkman formulation," Int. J. Heat Mass Transfer 39, 1363 (1996).

${ }^{34} \mathrm{~A}$. J. Chamkha and H. Al-Naser, "Double-diffusive convection in an inclined porous enclosure with opposing temperature and concentration gradients," Int. J. Therm. Sci. 40, 227 (2001).

${ }^{35}$ M. Bourich, A. Amahmid, and M. Hasnaoui, "Double diffusive convection in a porous enclosure submitted to cross gradients of temperature and concentration," Energy Convers. Manage. 45, 1655 (2004).

${ }^{36} \mathrm{~S}$. Akbal and F. Baytas, "Effects of non-uniform porosity on double diffusive natural convection in a porous cavity with partially permeable wall," Int. J. Therm. Sci. 47, 875 (2008).

${ }^{37}$ A. J. Chamkha and A. Al-Mudhaf, "Double-diffusive natural convection in inclined porous cavities with various aspect ratios and temperature-dependent heat source or sink," Heat Mass Transfer 44, 679 (2008).

${ }^{38}$ A. Mchirgui, N. Hidouri, M. Magherbi, and A. B. Brahim, "Second law analysis in double diffusive convection through an inclined porous cavity," Comput. Fluids 96, 105 (2014). 
${ }^{39}$ A. A. Mohamad, R. Bennacer, and J. Azaiez, "Double diffusion natural convection in a rectangular enclosure filled with binary fluid saturated porous media: The effect of lateral aspect ratio," Phys. Fluids 16, 184 (2004).

${ }^{40} \mathrm{P}$. Bera, S. Pippal, and A. K. Sharma, "A thermal non-equilibrium approach on double-diffusive natural convection in a square porous-medium cavity," Int. J. Heat Mass Transfer 78, 1080 (2014).

${ }^{41}$ S. C. Hirata, B. Goyeau, and D. Gobin, "Stability of thermosolutal natural convection in superposed fluid and porous layers," Transp. Porous Media 78, 525 (2009).

${ }^{42} \mathrm{GH}$. R. Kefayati, "Simulation of double diffusive natural convection and entropy generation of power-law fluids in an inclined porous cavity with Soret and Dufour effects (Part I: Study of fluid flow, heat and mass transfer)," Int. J. Heat Mass Transfer 94, 539 (2016).

${ }^{43} \mathrm{GH}$. R. Kefayati, "Simulation of double diffusive natural convection and entropy generation of power-law fluids in an inclined porous cavity with Soret and Dufour effects (Part II: Entropy generation)," Int. J. Heat Mass Transfer 94, 582 (2016)

${ }^{44}$ Q. Y. Zhu, Y. J. Zhuang, and H. Z. Yu, "Three-dimensional numerical investigation on thermosolutal convection of power-law fluids in anisotropic porous media," Int. J. Heat Mass Transfer 104, 897 (2017).

${ }^{45}$ D. M. Stefanescu, Science and Engineering of Casting Solidification (Springer, New York, 2002).

${ }^{46}$ T. J. Smith, Modelling the Flow and Solidification of Metals (Martinus Nijhoff Publishers, Boston, 1987).

${ }^{47} \mathrm{D}$. Vola, L. Boscardin, and J. Latche, "Laminar unsteady flows of Bingham fluids: A numerical strategy and some benchmark results," J. Comput. Phys. 187, 441 (2003).

${ }^{48} \mathrm{O}$. Turan, N. Chakraborty, and R. J. Poole, "Laminar natural convection of Bingham fluids in a square enclosure with differentially heated side walls," J. Non-Newtonian Fluid Mech. 165, 901 (2010).

${ }^{49} \mathrm{O}$. Turan, R. J. Poole, and N. Chakraborty, "Aspect ratio effects in laminar natural convection of Bingham fluids in rectangular enclosures with differentially heated side walls," J. Non-Newtonian Fluid Mech. 166, 208 (2011).

${ }^{50} \mathrm{O}$. Turan, N. Chakraborty, and R. J. Poole, "Laminar Rayleigh-Bénard convection of yield stress fluids in a square enclosure," J. Non-Newtonian Fluid Mech. 171-172, 83 (2012).

${ }^{51}$ A. Davaille, B. Gueslin, A. Massmeyer, and E. Di Giuseppe, "Thermal instabilities in a yield stress fluid: Existence and morphology," J. Non-Newtonian Fluid Mech. 193, 144 (2013).

${ }^{52}$ A. Massmeyer, E. Di Giuseppe, A. Davaille, T. Rolf, and P. J. Tackley, "Numerical simulation of thermal plumes in a Herschel-Bulkley fluid," J. Non-Newtonian Fluid Mech. 195, 32 (2013).
${ }^{53}$ I. Karimfazli and I. A. Frigaard, "Natural convection flows of a Bingham fluid in a long vertical channel," J. Non-Newtonian Fluid Mech. 201, 39 (2013).

${ }^{54}$ R. R. Huilgol and GH. R. Kefayati, "Natural convection problem in a Bingham fluid using the operator-splitting method," J. Non-Newtonian Fluid Mech. 220, 22 (2015).

${ }^{55}$ I. Karimfazli, I. A. Frigaard, and A. Wachs, "Thermal plumes in viscoplastic fluids: Flow onset and development," J. Fluid Mech. 787, 474 (2016).

${ }^{56}$ I. Karimfazli and I. A. Frigaard, "Flow, onset and stability: Qualitative analysis of yield stress fluid flow in enclosures," J. Non-Newtonian Fluid Mech. 238, 224 (2016).

${ }^{57}$ A. Dutta, A. K. Gupta, G. Mishra, and R. P. Chhabra, "Effect of fluid yield stress and of angle of tilt on natural convection from a square bar in a square annulus," Comput. Fluids 160, 138 (2018).

${ }^{58} \mathrm{GH}$. R. Kefayati and H. Tang, "Lattice Boltzmann simulation of viscoplastic fluids on natural convection in an inclined enclosure with inner cold circular/elliptical cylinders (Part I: One cylinder)," Int. J. Heat Mass Transfer 123, 1138 (2018).

${ }^{59} \mathrm{GH}$. R. Kefayati and H. Tang, "Lattice Boltzmann simulation of viscoplastic fluids on natural convection in an inclined enclosure with inner cold circular/elliptical cylinders (Part II: Two cylinders)," Int. J. Heat Mass Transfer 123, 1163 (2018).

${ }^{60} \mathrm{GH}$. R. Kefayati and H. Tang, "Lattice Boltzmann simulation of viscoplastic fluids on natural convection in an inclined enclosure with inner cold circular/elliptical cylinders (Part III: Four cylinders)," Int. J. Heat Mass Transfer 123, 1182 (2018).

${ }^{61}$ S. Yigit, R. J. Poole, and N. Chakraborty, "Effects of aspect ratio on natural convection of Bingham fluids in rectangular enclosures with differentially heated horizontal walls heated from below," Int. J. Heat Mass Transfer $\mathbf{8 0}$, 727 (2015).

${ }^{62}$ GH. R. Kefayati, "Double-diffusive natural convection and entropy generation of Bingham fluid in an inclined cavity," Int. J. Heat Mass Transfer 116, 762 (2018).

${ }^{63} \mathrm{GH}$. R. Kefayati and H. Tang, "Mesoscopic simulation of double-diffusive natural convection and entropy generation of Bingham fluid in an open cavity," Eur. J. Mech.: B/Fluids 69, 1 (2018).

${ }^{64}$ E. C. Bingham, Fluidity and Plasticity (McGraw-Hill, New York, 1922).

${ }^{65}$ T. C. Papanastasiou, "Flow of materials with yield," J. Rheol. 31, 385 (1987).

${ }^{66}$ Y. Dimakopoulos, M. Pavlidis, and J. Tsamopoulos, "Steady bubble rise in Herschel-Bulkley fluids and comparison of predictions via the augmented Lagrangian method with those via the Papanastasiou model," J. Non-Newtonian Fluid Mech. 200, 34 (2013). 